\title{
Flight Envelope Calculation of a Hypersonic Vehicle Using a First Principles-Derived Model
}

\author{
Derek J. Dalle*, \\ University of Michigan, Ann Arbor, MI 48109 \\ Michael A. Bolender ${ }^{\dagger}$ \\ U.S. Air Force Research Laboratory, Wright-Patterson Air Force Base, Ohio, 45433 \\ Sean M. Torrez $\ddagger$ James F. Driscoll ${ }^{\S}$ \\ University of Michigan, Ann Arbor, MI 48109
}

\begin{abstract}
Steady, level flight for an air-breathing hypersonic vehicles requires balancing intricate couplings among the engine, lifting surfaces, and control effectors. A newly developed fundamental model is used to determine the range of flight Mach numbers and altitudes at which this balance can be obtained. The hypersonic vehicle was developed specifically for flight dynamics evaluations, and the model can calculate the net forces and moments on a three-dimensional vehicle in less than ten seconds using a single $2.6 \mathrm{GHz}$ processor. The propulsive model includes complex physics such as wave interactions, fuel mixing, and finite-rate chemistry. This type of model requires less computational resources than a model based on computational fluid dynamics and provides a more accurate characterization of the flight envelope than simplified models could.
\end{abstract}

\section{Nomenclature}

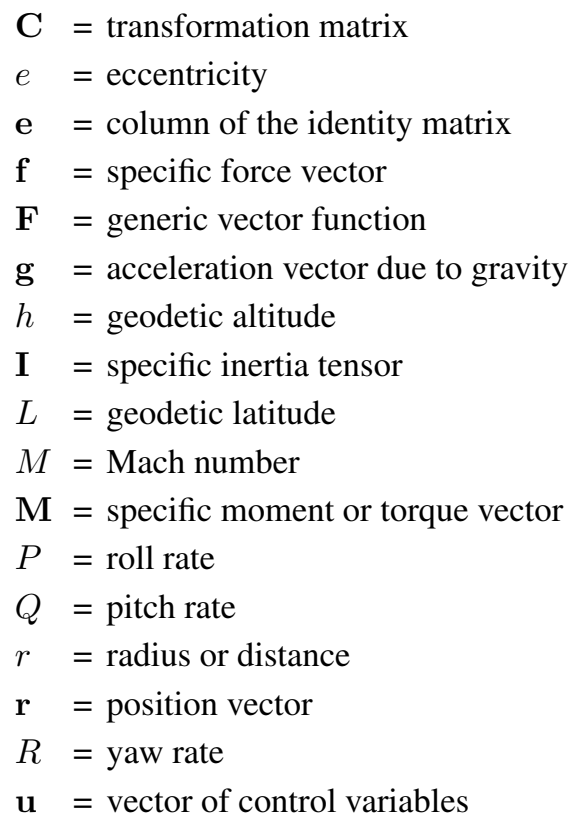

$$
\begin{aligned}
U & =\text { velocity scalar } \\
\mathbf{v} & =\text { velocity vector } \\
V & =\text { velocity scalar } \\
W & =\text { velocity scalar } \\
x & =\text { reference axis or coordinate } \\
\mathbf{x} & =\text { vector of state variables } \\
y & =\text { reference axis or coordinate } \\
\mathbf{y} & =\text { vector of output variables } \\
z & =\text { reference axis or coordinate } \\
\alpha & =\text { angle of attack } \\
\beta & =\text { sideslip angle } \\
\gamma & =\text { flight path angle } \\
\delta & =\text { deflection angle } \\
\eta & =\text { small step size } \\
\Theta & =\text { pitch angle } \\
\lambda & =\text { longitude } \\
\boldsymbol{\xi} & =\text { independent trim variables }
\end{aligned}
$$

\footnotetext{
${ }^{*}$ Graduate Research Assistant, Department of Aerospace Engineering, AIAA Member, dalle@umich . edu

${ }^{\dagger}$ Aerospace Engineer, U.S. Air Force Research Laboratory, 2210 Eighth Street, Suite 21. Associate Fellow AIAA.

$\ddagger$ Graduate Research Assistant, Department of Aerospace Engineering, AIAA Member, smtorrez@umich. edu

$\S$ Professor, Department of Aerospace Engineering, AIAA Fellow, jamesfd@umich . edu
} 


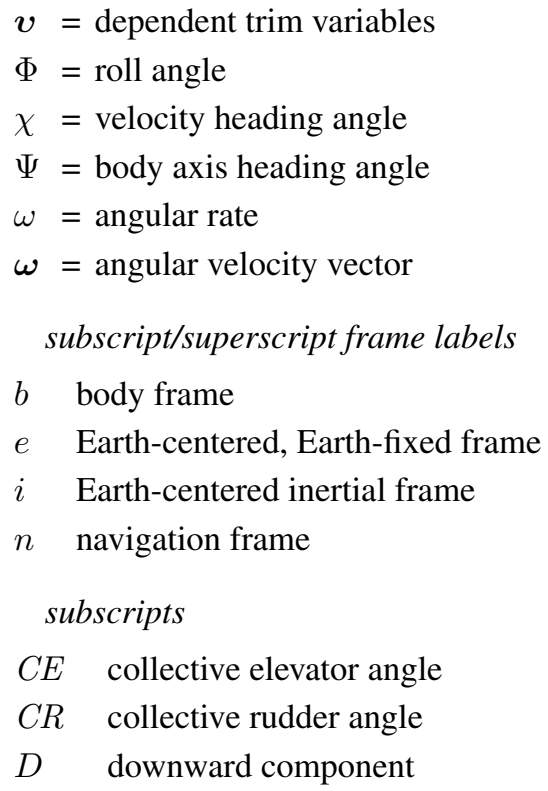

\begin{tabular}{ll}
$D E$ & difference between elevators \\
$D R$ & difference between rudders \\
$E$ & east component \\
$E R$ & fuel-air equivalence ratio \\
$j$ & vector component index \\
$k$ & vector component index \\
$N$ & north component \\
$Q$ & value at equator \\
$t$ & total velocity \\
& \\
\multicolumn{1}{c}{ modifiers } \\
$\dot{a}$ & time derivative \\
$\ddot{a}$ & second time derivative \\
$\bar{a}$ & value at trim \\
$\Delta a$ & small perturbation \\
$a^{T}$ & matrix transpose
\end{tabular}

\section{Introduction}

In addition to maximizing fuel efficiency, ensuring adequate thrust and lift, and limiting heat transfer, a hypersonic vehicle must be able to balance all of the forces acting on it such that the velocity and attitude of the vehicle remain constant. Using this as a guideline, we define the flight envelope as the set of flight Mach numbers, $M$, and altitudes, $h$, at which the vehicle is able to meet these conditions for steady, level flight. In order to simulate this balanced, or trimmed, flight condition, the forces on the vehicle must be calculated for many combinations of input variables, and a model is required that can evaluate the performance of the entire vehicle in a few seconds or less.

Previous models capable of analyzing trim of a full air-breathing hypersonic vehicle include are three-degree-offreedom longitudinal models by Schöttle and Hillesheimer [1], Chavez and Schmidt [2], Bolender and Doman [3], and Parker et al. [4] and a six-degree-of-freedom model by Frendreis and Cesnik [5]. The primary focus of the present work is to add more accurate modeling the vehicle. For example, we now account for complex phenomena such as wave interactions, finite-rate chemistry, and fuel mixing. Simpler models may be accurate for a narrow range of conditions at which a good vehicle design can ensure proper compression and fuel mixing, but factors such as wave interactions and poor fuel mixing have been shown to play a significant role for a vehicle with a wide flight envelope $[6,7]$.

The present model, known as Michigan/AFRL Scramjet Trim, or MASTrim, incorporates a three-dimensional vehicle geometry and includes a higher-fidelity one-dimensional model of the propulsive system than what was previously used. Although it lacks a model for vehicle deformations, we can accommodate the effects of flexibility by superimposing the mode shapes of the vehicle onto the geometry. We used version 2.2 of this code to obtain the data in this paper. The code is designed for analysis of vehicles that are designed with planar inlets-such as the X-43, X-51, and the National Aerospace Plane. An example vehicle is shown in Fig. 1, which is the vehicle used in the present analysis. The package also includes a tool to produce similar vehicles based on 62 design parameters. Example parameters include vehicle length, stabilator location, and engine width.

In our view, the advantage to using this type of model is that it has the capability to model the performance of a hypersonic vehicle over a wide range of conditions with relatively little computation. For example, previous work, such as $[6,8]$, has shown that a very small change in angle of attack or Mach number can on some occasions cause a large change in the inlet performance. An inlet model that does not account for wave interactions would have no way to predict this performance drop. It is possible to design inlets that reduce this effect, [9], and as a result a simplified analysis may be accurate for initial analysis of on-design performance. However, a more capable model is needed to develop such an inlet design.

While determining if steady, level flight is possible for a certain condition, our model does not account for structural and heating considerations. As a result, the actual flight envelope of the hypersonic vehicle would be a subset of the ones presented here. In the process of determining the flight envelope, we calculate trimmed flight conditions across the entire envelope, which provides an operating map for the vehicle that gives approximate control inputs needed to fly at a given combination of speed and altitude. 


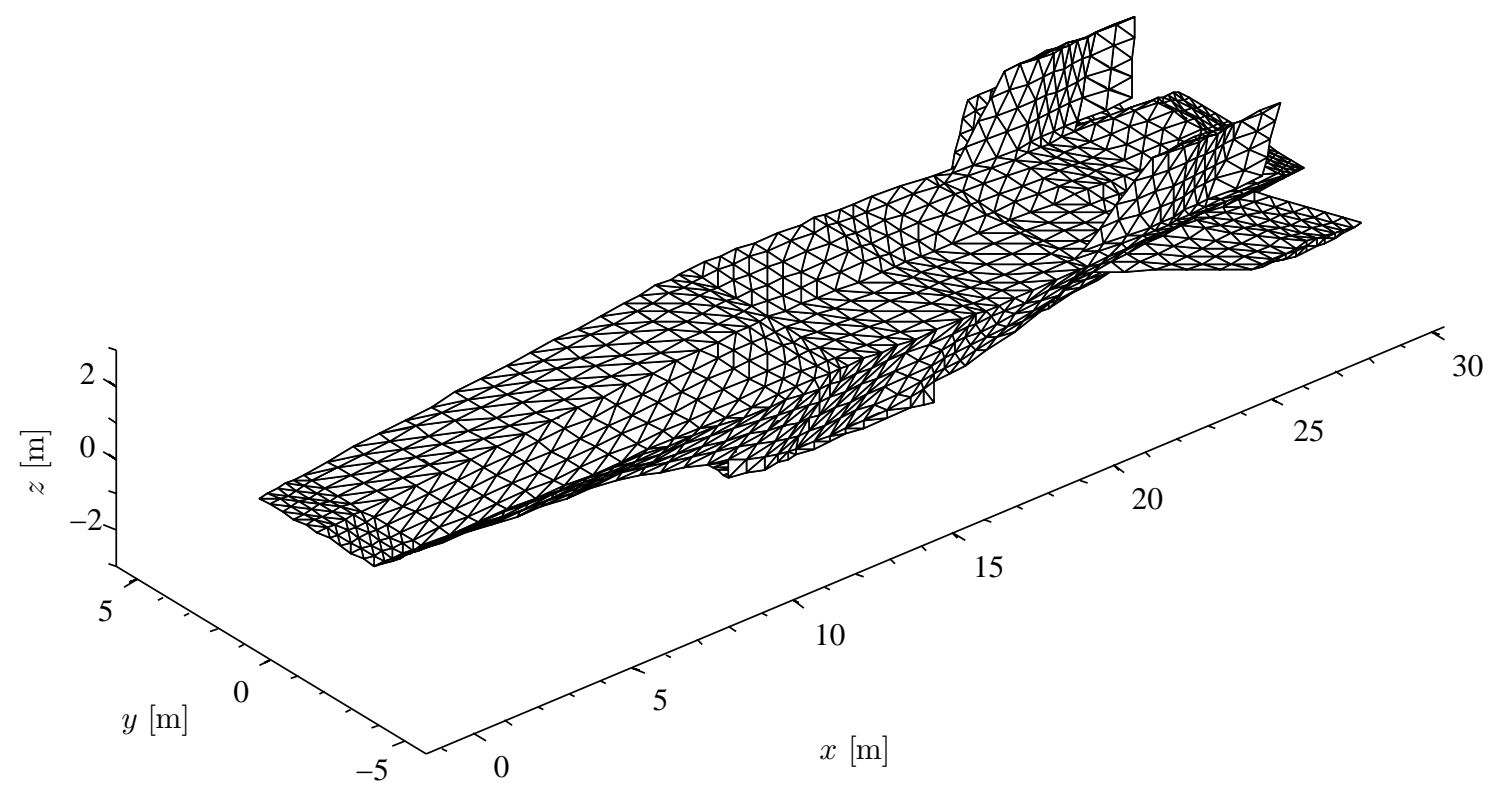

Figure 1. Isometric view of example vehicle

We use a rotating, elliptical model for Earth, which means that the trimmed flight condition will be dependent on latitude and heading angle. In this analysis we are able to investigate how the operating map is affected by a large number of other parameters. For example, we are able to see if changing the mass of fuel present in the vehicle affects the trim conditions, which we expect will happen since the vehicle will need higher lift. We also compare flight at different latitudes and compare climbing flight to level flight.

\section{Hypersonic Vehicle Model}

The vehicle model consists of separate models for the inlet, isolator, combustor, nozzle, and the external aerodynamic components that are carefully integrated to ensure that the flight dynamics of the vehicle are realistically captured. For modeling purposes, the vehicle is split into propulsive and nonpropulsive sections, which is consistent with standard convention. The engine model is responsible for the inlet, isolator, combustor, and nozzle, and the external aerodynamic model is responsible for the remaining surfaces. In Fig. 2, the surfaces that are considered to be part of the engine model are shown in red. This modeling approach is limited to vehicles that are similar to an X-43. In particular, it is limited to vehicles for which the inlet and nozzle can be approximated reasonably as two-dimensional flow, but the model does neglect three-dimensional flow effects, such as lateral spillage.

\section{A. Engine Model}

The engine model is the primary component of MASTrim, and as a result, it has its own acronym. We refer to the engine model as the Michigan/AFRL Scramjet In Vehicle, or MASIV, code. The MASIV engine model is designed to have enough fidelity to capture the important physics of the combustion process while still being able to run in a few seconds or less on a single personal computer. In a true reduced-order model approach, a large amount of computationally expensive analysis is needed for each new vehicle design, and afterward analysis can be done rapidly [10]. Rather than taking this kind of approach, we have developed the model as a direct, low-order physics model. As a result, the MASIV model can be used to design a new vehicle geometry in just a few seconds, and the first trimmed flight condition can be calculated in only one or two minutes after that.

Of course, speed is not the only concern in the MASIV model. Actually, the primary contribution that the model provides is a higher level of complexity and fidelity than what has been previously available for control or flight dynamics studies during the conceptual design phase of the vehicle design process. For example, we found that 


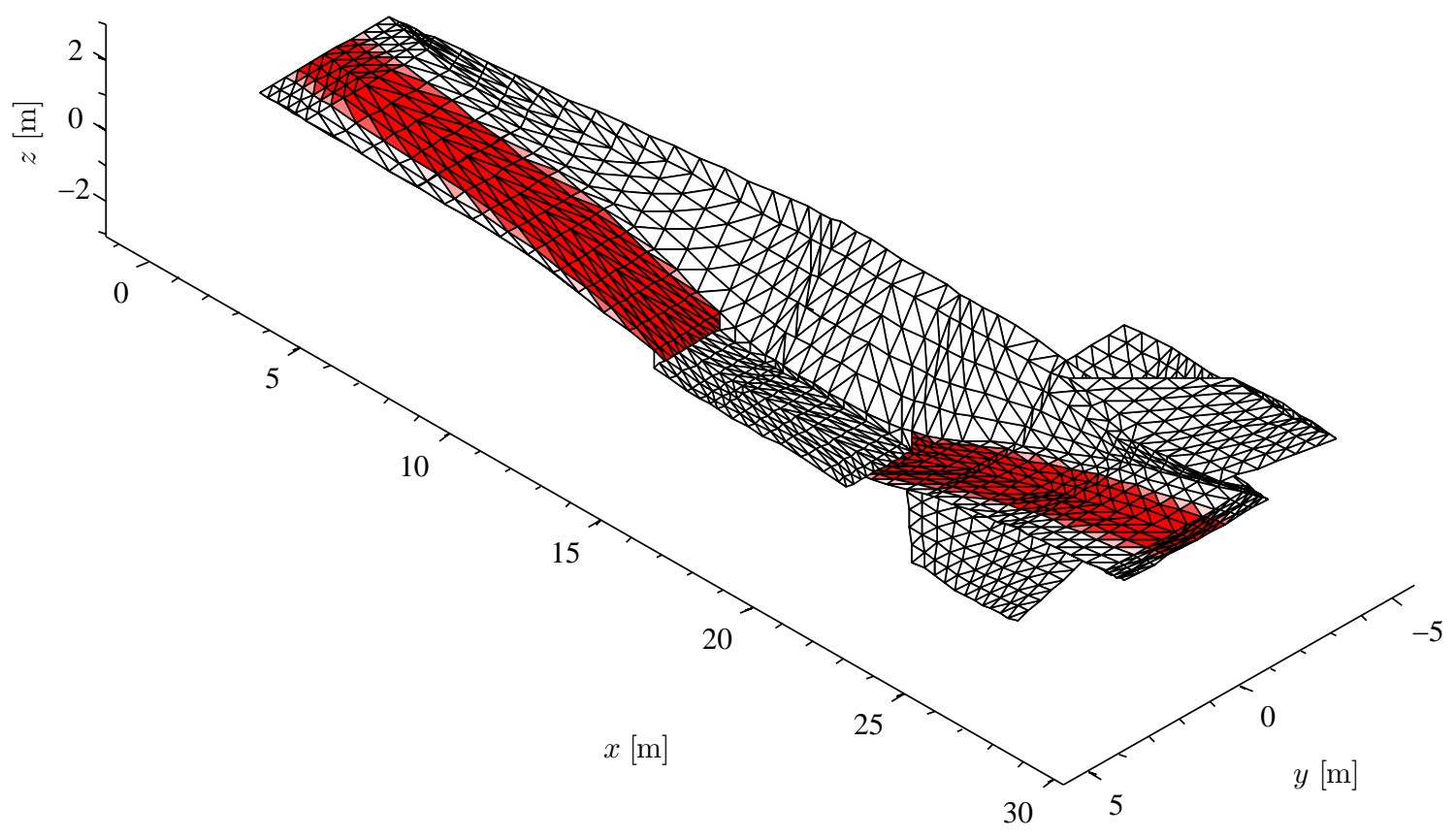

Figure 2. View of the vehicle from below with propulsive surfaces in red

accounting for interactions between shock waves in the inlet affects the performance by a substantial amount for offdesign flight conditions [6]. As a result, we developed a method to design inlets that is more robust with respect to a wider range of flight conditions [9]. In another example, we found that inadequate fuel-air mixing in the combustor caused a dramatic decrease in fuel efficiency [7].

The inlet and nozzle use the same underlying physics to predict performance. This model is called Supersonic Aerodynamic Model Using Riemann Interactions (SAMURI), and it creates a solution that is similar in appearance to two-dimensional computational fluid dynamics (CFD). Instead of creating a grid and solving for the flow conditions at each point in the grid, SAMURI works by tracking the positions of the vehicle surfaces, shock waves, expansion waves, and contact discontinuities. It begins at the most upstream coordinate in the flow region and only computes wave and post-wave properties at leading edges, vertices of the vehicle surface, points where at least two waves interact, or points where at least one wave interacts with the vehicle surface. This approach is limited to two-dimensional surfaces that consist of a series of flat plates, although a curved surface potentially can be modeled in this way by splitting the curve into a finite number of flat sections. Also, since this approach assumes that information cannot propagate upstream, it is limited to supersonic flows. The output of this model is a collection of regions for which the boundaries are all either waves or the surface of the vehicle itself. In each of these regions, the flow properties (pressure, temperature, etc.) are constant.

After the waves and flow solution have been computed, net forces are calculated using a control volume analysis. This requires tracing streamlines (upstream in the case of the inlet and downstream in the case of the nozzle). Viscous forces are taken into account in the form of a boundary layer, which is modeled as a momentum thickness. The flow in the boundary layer has a thermodynamic state (pressure, density, temperature) but no flow velocity. The height of the momentum layer is defined such that a control volume analysis predicts drag using these assumptions in a way that is consistent with conservation of mass, momentum, and energy [11]. The actual value of the momentum thickness at each point is calculated using the Van Driest II method, [12], which is commonly used in the part of the design process where this code is intended to apply. An example solution is shown in Fig. 3.

The combustion code marches the one-dimensional flow conservation equations from the beginning of the combustor to the end of the internal nozzle. All flow states are allowed to vary in the downstream axial direction. To keep 


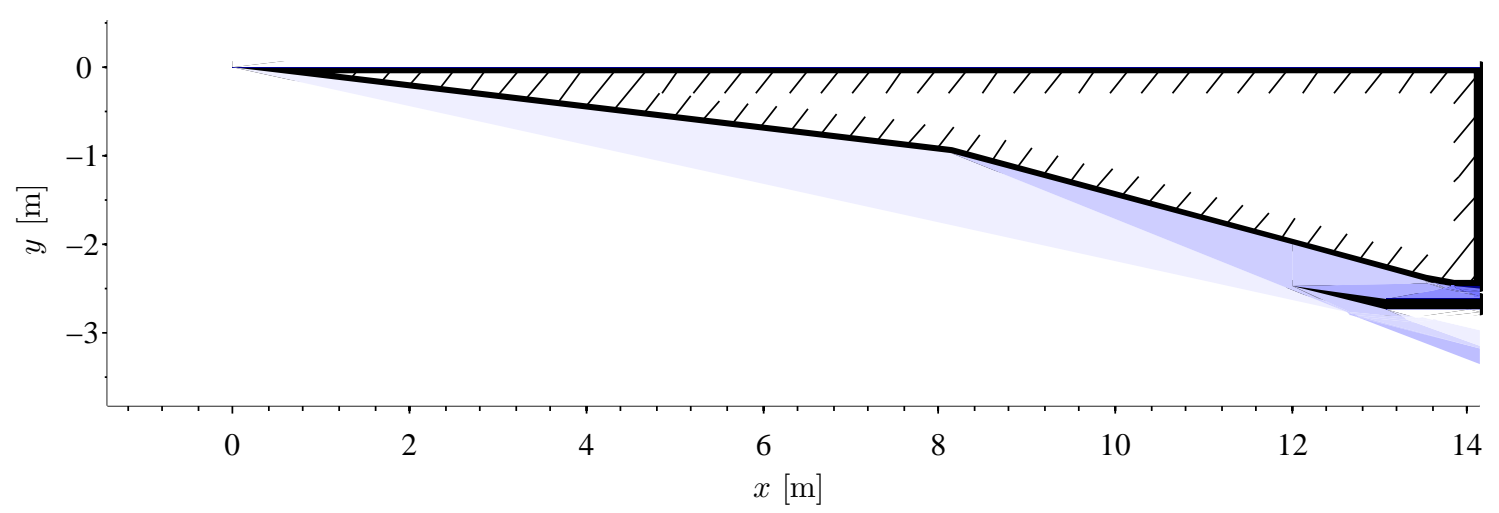

a) Full inlet

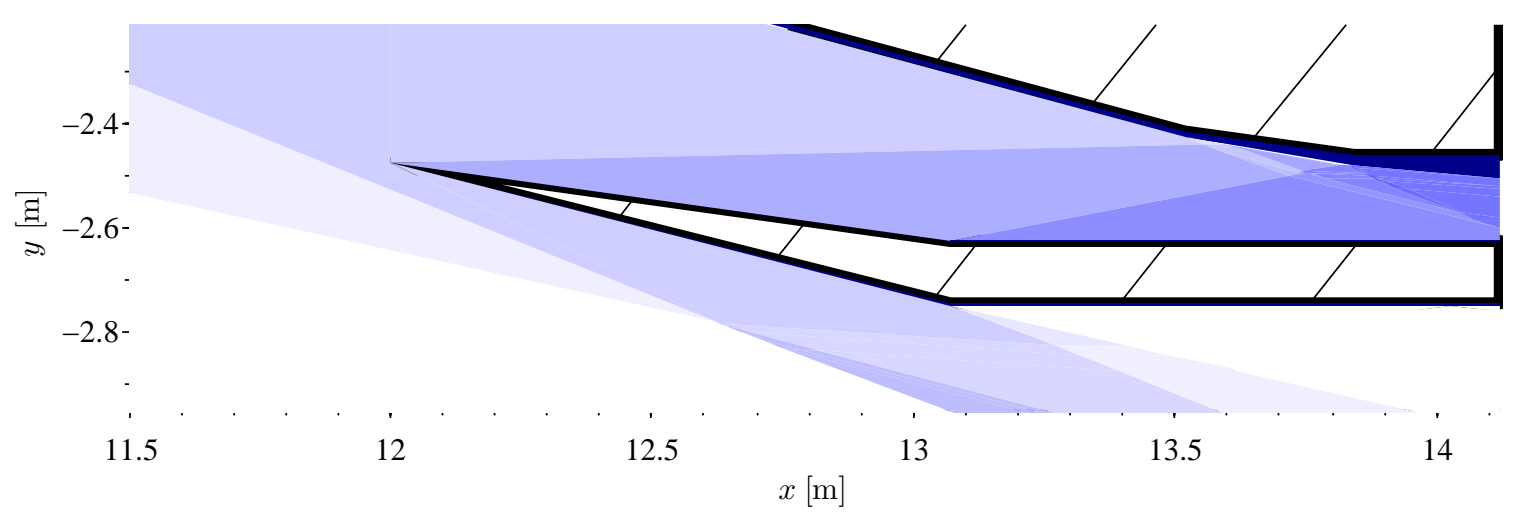

b) Zoomed in

Figure 3. Temperature plot for an example inlet for trimmed flight at Mach 8 and an altitude of $26 \mathbf{~ k m}$

the model one-dimensional, only derivatives with respect to the axial coordinate are considered. Some quantities, such as jet spreading and mixing, vary in the transverse directions, but they may only vary algebraically such that their evolutions do not depend on the information propagating downstream. This allows, to some degree, for a model of fuel-air mixing without introducing the computational expense required for a two- or three-dimensional flow solution.

When the engine is operating in scram mode, the flow velocity is higher than the local speed of sound throughout the combustor. However, the heat release in the combustor tends to drive the local Mach number to 1, and if enough fuel is added, the flow through the combustor eventually becomes subsonic (thermally choked) at some point. When this happens, the entire combustor switches to ram mode, and the operation changes significantly. Although we have developed a model for ram-mode flight, it has not been tested adequately for inclusion in this paper, and the results in this discussion are the scram-mode flight envelope.

The result of this analysis is a one-dimensional profile for density, pressure, temperature, flow velocity, and species mass fractions. These results are shown in Fig. 4 for trimmed flight at Mach 8 and an altitude of $26 \mathrm{~km}$. Using this data, we calculate the net forces and moments on the combustor and isolator. Actually, the forces and moments are calculated per unit width since the engine model is two-dimensional. The force on the engine is then the sum of the forces on each component times the width of the engine.

\section{B. External Aerodynamics}

The remaining surfaces (those that are not red in Fig. 2) are handled by a simple shock/expansion method with a boundary layer correction to account for viscous drag. For each small triangular surface, the freestream velocity is projected onto the surface. The angle between the freestream and this projected vector is then used to compute the thermodynamic properties behind either a shock or a Prandtl-Meyer expansion. The projected vector is also used to compute the boundary layer properties using the Van Driest II method [12]. 


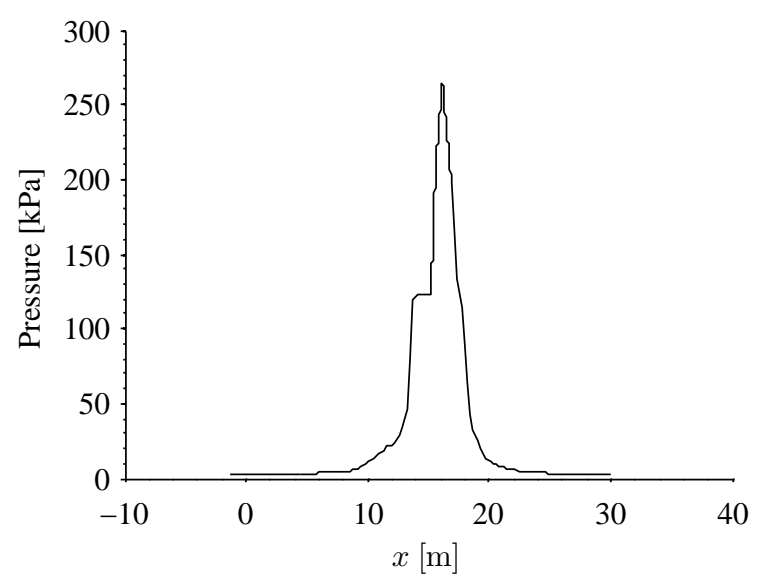

a) Pressure

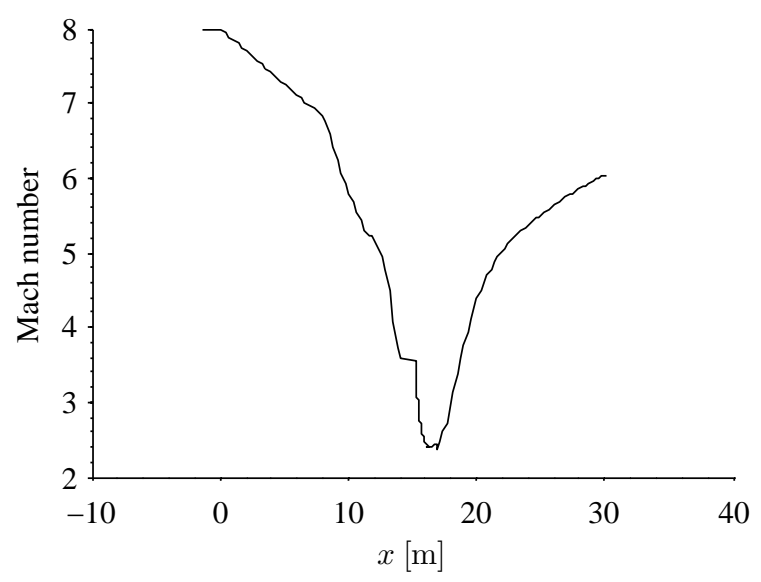

c) Mach number

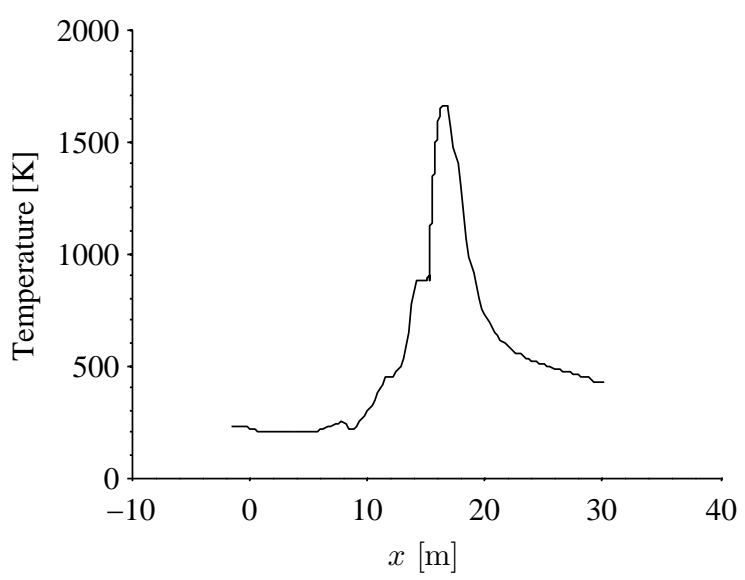

b) Temperature

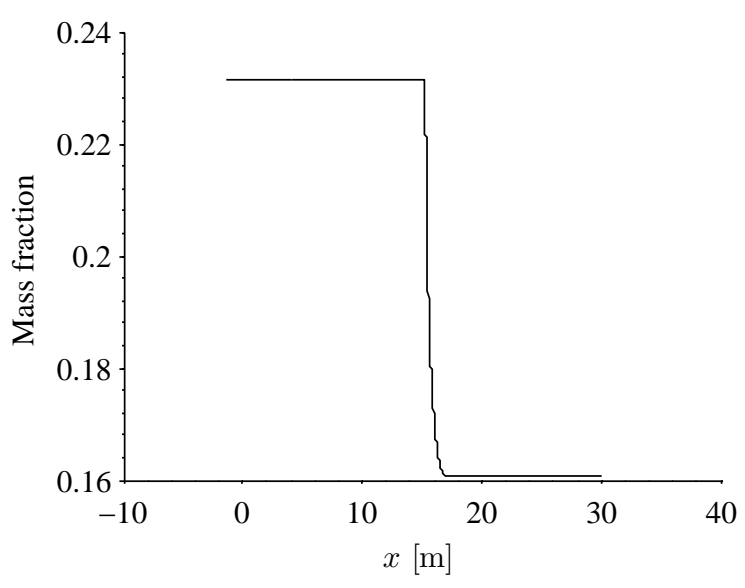

d) Mass fraction of $\mathrm{O}_{2}$

Figure 4. One-dimensional profiles for trimmed flight condition at Mach 8 and an altitude of $26 \mathrm{~km}$ 
For any surface that is not along a leading edge, the boundary layer already exists on the upstream surfaces. Considering the boundary layer to start over on each surface would result in a considerable overestimate of viscous drag. To account for this, a momentum thickness is calculated for each edge. For each surface, the momentum thickness at the upstream edge(s) is used to calculate the momentum thickness at the downstream edge(s), which is then passed on to the next surface. This model provides a first estimate of the viscous drag on the exterior of the vehicle, but it does not account for shock-boundary layer interactions. This differs from the boundary model used in the inlet and nozzle, in which the deflection angle caused by the boundary layer itself is considered part of the deflection angle that determines the strength of the shock.

\section{Equations of Motion}

For hypersonic vehicles, the velocities and altitude are high enough that the assumption a flat Earth is no longer valid. As a result, we employ a model that is consistent with a rotating WGS84 Earth [13, 14]. For example, an aircraft flying east at a constant altitude above the equator would require a lift force that is about $13 \%$ less than what would be predicted when using the flat-Earth equations of motion.

The output of the hypersonic vehicle model is a net specific force (i.e. net force divided by vehicle mass) and net specific moment in the body frame, which we write

$$
\begin{aligned}
\mathbf{f}^{b} & =\mathbf{f}^{b}(\mathbf{x}, \mathbf{u}) \\
\mathbf{M}^{b} & =\mathbf{M}^{b}(\mathbf{x}, \mathbf{u})
\end{aligned}
$$

Here $\mathbf{x}$ is a vector of state variables, and $\mathbf{u}$ is a vector of control variables. Since these forces and moments are calculated in a frame fixed to the body of the vehicle, we will write the equations of motion in this coordinate system. However, since this frame is not an inertial frame, we cannot calculate the accelerations by just adding up all the forces in the body frame and dividing by the mass. Instead, we must apply the Coriolis transport theorem to account for the rotation of the body-fixed frame.

\section{A. Coordinate Systems}

In order to apply Newton's second law directly, we need an inertial frame. For our purposes, this will be the Earthcentered inertial (ECI) frame, and we label it the $i$-frame.

$$
\ddot{\mathbf{r}}_{i b}^{i}=\mathbf{f}^{i}+\mathbf{g}^{i}
$$

We also see the need to be able to transform a vector from one coordinate system to another. We do this with a transformation matrix, for which we use the following notation.

$$
\mathbf{f}^{i}=\mathbf{C}_{b}^{i} \mathbf{f}^{b}
$$

Here the $b$ denotes the body frame, which is attached to the vehicle such that the $x$-axis points forward, the $y$-axis points to the right, and the $z$-axis points downward. This notation is saying that multiplying a vector that is resolved in the $b$-frame by $\mathbf{C}_{b}^{i}$ on the left results in the same vector resolved in the $i$-frame.

The notation $\mathbf{r}_{i b}^{i}$ requires more explanation. The $\mathbf{r}_{i b}$ part refers to the vector from the origin of the $i$-frame (the center of the Earth) to the origin of the $b$-frame (the center of mass in our case). The superscript $i$ means that the vector is written as a vector in the $i$ coordinate system.

Since the Earth is rotating, and the atmosphere more or less rotates with it (neglecting weather patterns), we describe the motion of the vehicle in a coordinate system where each point on the surface of the Earth has constant coordinates. For this purpose, we use the Earth-centered, Earth-fixed frame (ECEF), in which the $z^{e}$-axis points from the center of the Earth toward the north pole, the $x^{e}$-axis points toward the intersection of the equator and the prime meridian, and the $y^{e}$-axis completes a right-handed system. The angular velocity between the ECEF frame and the ECI frame is

$$
\boldsymbol{\omega}_{i e}^{e}=\left[\begin{array}{c}
0 \\
0 \\
\omega_{i e}
\end{array}\right] \quad \omega_{i e} \approx 7.292115 \times 10^{-5} \mathrm{rad} / \mathrm{s}
$$

To make the equations more intuitive, we introduce a coordinate system in which the $x$-axis points toward the local north, the $y$-axis points toward the local east, and the $z$-axis points locally down. This is called the navigation frame, 


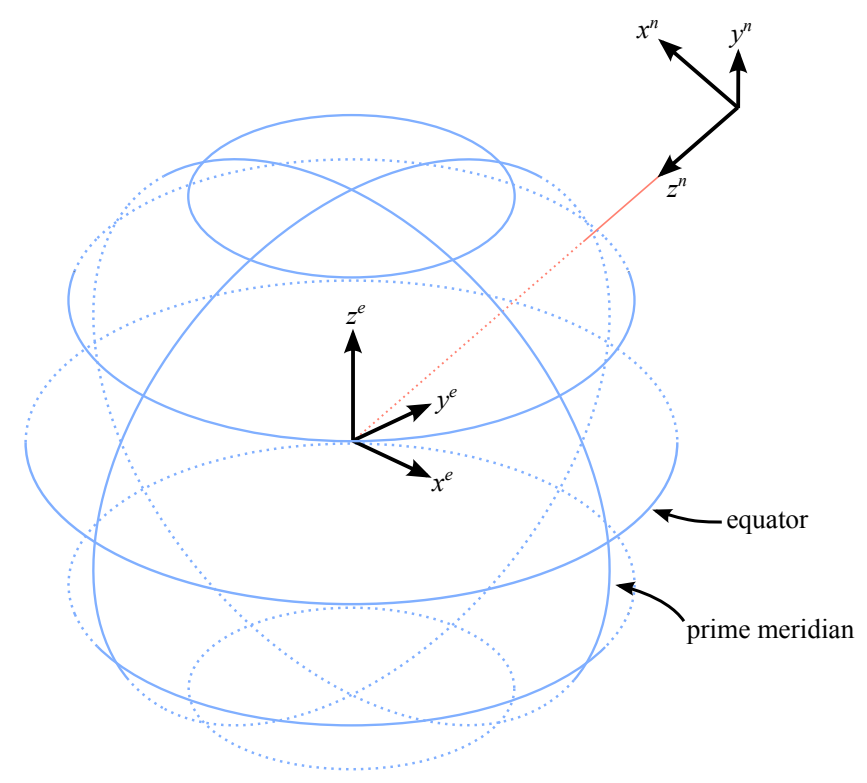

Figure 5. ECEF and navigation coordinate frames

and the transformation matrix from the navigation frame to the ECEF frame is

$$
\mathbf{C}_{n}^{e}=\left[\begin{array}{ccc}
-\sin L \cos \lambda & -\sin \lambda & -\cos L \cos \lambda \\
-\sin L \sin \lambda & \cos \lambda & -\cos L \sin \lambda \\
\cos L & 0 & -\sin L
\end{array}\right]
$$

The navigation frame is carried with the vehicle, so that the origin of the navigation frame is always located at the center of mass of the vehicle. However, the navigation frame does not rotate with the vehicle, so that, for example, the $z^{n}$ axis always points directly normal to the surface of the Earth. Figure 5 gives a visual demonstration of the relation between the ECEF and navigation coordinate frames for an example point. The components of the vehicle velocity in this frame are

$$
\mathbf{v}_{e b}^{n}=\mathbf{C}_{e}^{n} \dot{\mathbf{r}}_{e b}^{e}=\left[\begin{array}{c}
V_{N} \\
V_{E} \\
V_{D}
\end{array}\right]
$$

This equation shows an implicit assumption made in this notation; derivatives that are shown with a dot are always taken in the resolving frame. This means, for example, that $\mathbf{v}_{e b}^{n} \neq \dot{\mathbf{r}}_{e b}^{n}$. Also, the vehicle velocity and position are both always measured from the ECEF origin to the body origin, so the two subscripts will be dropped in the following references.

The final coordinate frame is the body frame, which was discussed previously and has the transformation matrix that carries the navigation frame to the body frame of

$$
\mathbf{C}_{n}^{b}=\left[\begin{array}{ccc}
1 & 0 & 0 \\
0 & \cos \Phi & \sin \Phi \\
0 & -\sin \Phi & \cos \Phi
\end{array}\right]\left[\begin{array}{ccc}
\cos \Theta & 0 & -\sin \Theta \\
0 & 1 & 0 \\
\sin \Theta & 0 & \cos \Theta
\end{array}\right]\left[\begin{array}{ccc}
\cos \Psi & \sin \Psi & 0 \\
-\sin \Psi & \cos \Psi & 0 \\
0 & 0 & 1
\end{array}\right]
$$

The components of the velocity are

$$
\mathbf{v}^{b}=\mathbf{C}_{e}^{b} \dot{\mathbf{r}}^{e}=\left[\begin{array}{c}
U \\
V \\
W
\end{array}\right]
$$




\section{B. The WGS84 Earth Model}

Instead of using a spherical Earth, we have chosen to use the WGS84 ellipsoidal reference datum, [13]. This is the same model used for the Global Positioning System (GPS), [14], and it thus provides an accurate reference for navigation. In this model, each curve of constant latitude is a circle, but each meridian is an ellipse with an eccentricity of

$$
e=0.0818191908426
$$

and a semi-major axis of

$$
r_{Q}=6378137 \mathrm{~m}
$$

One result of using an ellipsoidal Earth is the possibility of ambiguously defined latitude and altitude. In this paper, we use geodetic latitude, which has the property that moving normal to the reference ellipsoid does not change the latitude. As a result, the ECEF coordinates in terms of latitude, longitude, and altitude are

$$
\begin{aligned}
& x_{e n}^{e}=\left(\frac{r_{Q}}{\sqrt{1-e^{2} \sin ^{2} L}}+h\right) \cos L \cos \lambda \\
& y_{e n}^{e}=\left(\frac{r_{Q}}{\sqrt{1-e^{2} \sin ^{2} L}}+h\right) \cos L \sin \lambda \\
& z_{e n}^{e}=\left(\frac{r_{Q}\left(1-e^{2}\right)}{\sqrt{1-e^{2} \sin ^{2} L}}+h\right) \sin L
\end{aligned}
$$

We also need the rotation rate and the angular acceleration of the $n$-frame with respect to the $e$-frame, which are

$$
\boldsymbol{\omega}_{e n}^{n}=\left[\begin{array}{c}
\dot{\lambda} \cos L \\
-\dot{L} \\
-\dot{\lambda} \sin L
\end{array}\right] \quad \dot{\omega}_{e n}^{n}=\left[\begin{array}{c}
\ddot{\lambda} \cos L-\dot{\lambda} \dot{L} \sin L \\
-\ddot{L} \\
-\ddot{\lambda} \sin L-\dot{\lambda} \dot{L} \cos L
\end{array}\right]
$$

where

$$
\begin{array}{ll}
\dot{\lambda}=\frac{V_{E}}{r_{E}} & \ddot{\lambda}=\left(\frac{\dot{v}_{E}}{V_{E}}-\frac{\dot{r}_{E}}{r_{E}}\right) \dot{\lambda} \\
\dot{L}=\frac{V_{N}}{r_{N}} & \ddot{L}=\left(\frac{\dot{v}_{N}}{V_{N}}-\frac{\dot{r}_{N}}{r_{N}}\right) \dot{L}
\end{array}
$$

Here $r_{E}$ refers to the radius of curvature in the east-west direction and $r_{N}$ is the radius of curvature in the north-south direction. Their formulas are

$$
\begin{array}{ll}
r_{E}=\left(\frac{r_{Q}}{\sqrt{1-e^{2} \sin ^{2} L}}+h\right) \cos L & \dot{r}_{E}=-V_{N} \sin L-V_{D} \cos L \\
r_{N}=\frac{r_{Q}\left(1-e^{2}\right)}{\left(1-e^{2} \sin ^{2} L\right)^{3 / 2}}+h & \dot{r}_{N}=\frac{3}{2} \frac{\left(r_{N}-h\right) e^{2} \sin 2 L}{1-e^{2} \sin ^{2} L} \frac{V_{N}}{r_{N}}-V_{D}
\end{array}
$$

\section{Net Accelerations}

The equations of motion in the $n$-frame, which are often called the navigation equations, [14], are

$$
\dot{\mathbf{v}}^{n}=\left[\begin{array}{c}
\dot{V}_{N} \\
\dot{V}_{E} \\
\dot{V}_{D}
\end{array}\right]=\mathbf{C}_{b}^{n} \mathbf{f}^{b}+\mathbf{g}^{n}-\boldsymbol{\omega}_{i e}^{n} \times\left(\boldsymbol{\omega}_{i e}^{n} \times \mathbf{C}_{e}^{n} \mathbf{r}^{e}\right)-\left(2 \boldsymbol{\omega}_{i e}^{n}+\boldsymbol{\omega}_{e n}^{n}\right) \times \mathbf{v}^{n}
$$

A simple application of the transport theorem provides the net accelerations in the body frame.

$$
\dot{\mathbf{v}}^{b}=\left[\begin{array}{c}
\dot{U} \\
\dot{V} \\
\dot{W}
\end{array}\right]=\mathbf{f}^{b}+\mathbf{C}_{n}^{b}\left(\mathbf{g}^{n}-\boldsymbol{\omega}_{i e}^{n} \times\left(\boldsymbol{\omega}_{i e}^{n} \times \mathbf{C}_{e}^{n} \mathbf{r}^{e}\right)-\left(2 \boldsymbol{\omega}_{i e}^{n}+\boldsymbol{\omega}_{e n}^{n}+\mathbf{C}_{b}^{n} \boldsymbol{\omega}_{n b}^{b}\right) \times \mathbf{v}^{n}\right)
$$


The angular equations of motion are derived in a similar fashion. The resulting equations are

$$
\dot{\boldsymbol{\omega}}_{n b}^{b}=\left(\mathbf{I}^{b}\right)^{-1}\left(\mathbf{M}^{b}-\boldsymbol{\omega}_{i b}^{b} \times \mathbf{I}^{b} \boldsymbol{\omega}_{i b}^{b}\right)-\mathbf{C}_{n}^{b}\left(\dot{\boldsymbol{\omega}}_{e n}^{n}+\boldsymbol{\omega}_{i n}^{n} \times \mathbf{C}_{b}^{n} \boldsymbol{\omega}_{n b}^{b}+\boldsymbol{\omega}_{i e}^{n} \times \boldsymbol{\omega}_{e n}^{n}\right)
$$

where

$$
\begin{aligned}
\boldsymbol{\omega}_{i n}^{n} & =\boldsymbol{\omega}_{i e}^{n}+\boldsymbol{\omega}_{e n}^{n} \\
\boldsymbol{\omega}_{i b}^{b} & =\mathbf{C}_{n}^{b} \boldsymbol{\omega}_{i n}^{n}+\boldsymbol{\omega}_{n b}^{b}
\end{aligned}
$$

and $\mathbf{I}^{b}$ is the inertia tensor divided by the total mass of the vehicle. In our model, we assume that changes in the mass of the vehicle simply change the uniform density of the entire vehicle volume, which is a first approximation used before the mass properties of the vehicle are really established. As a result, $\mathbf{I}^{b}$ is independent of the vehicle mass in this simple model. The components of the body angular velocity and its derivative are

$$
\boldsymbol{\omega}_{n b}^{b}=\left[\begin{array}{c}
P \\
Q \\
R
\end{array}\right] \quad \dot{\boldsymbol{\omega}}_{n b}^{b}=\left[\begin{array}{c}
\dot{P} \\
\dot{Q} \\
\dot{R}
\end{array}\right]
$$

\section{Trim}

For the purposes of finding a trimmed flight condition, we combine the equations of motion into the form

$$
\mathbf{y}=\mathbf{F}(\mathbf{x}, \mathbf{u})
$$

The output vector, y, consists of the velocity and angular velocity derivatives, $\dot{U}, \dot{V}, \dot{W}, \dot{P}, \dot{Q}$, and $\dot{R}$. Finding a trimmed flight condition, then, reduces to picking a value of $\mathbf{y}$ (usually $\mathbf{0}$ ), and finding values of $\mathbf{x}$ and $\mathbf{u}$ that satisfy Eq. (26). Typically, we want to pick values for some of the state variables and find values for the remaining state variables and the control variables. Using these concepts, we can rewrite Eq. (26) as

$$
\mathbf{y}=\mathbf{F}(\boldsymbol{\xi}, \boldsymbol{v})
$$

where $\boldsymbol{\xi}$ is a vector of the independent state variables, and $\boldsymbol{v}$ contains the dependent state variables and the control variables. In this discussion, we consider the independent state variables to be those describing the position, velocity, and angular velocity. The dependent variables include the orientation of the vehicle and the various control variables.

For this paper, the independent variables are

$$
\boldsymbol{\xi}=\left[\begin{array}{lllllllll}
L & \lambda & h & M & \gamma & \chi & P & Q & R
\end{array}\right]^{T}
$$

and the dependent variables are

$$
\boldsymbol{v}=\left[\begin{array}{llllllll}
\alpha & \beta & \Phi & \delta_{E R} & \delta_{C E} & \delta_{D E} & \delta_{C R} & \delta_{D R}
\end{array}\right]^{T}
$$

where $\delta_{C E}$ is the average of the left and right elevator deflection angles (defined so that a positive deflection moves the trailing edge down) and $\delta_{D E}$ is the deflection angle of the right elevator minus the deflection angle of the left elevator. For simplicity, we also make the assumption that the sideslip angle, $\beta$, and the differential rudder angle, $\delta_{D R}$, are both zero. This is convenient because it makes Eq. (27) a system of equations with six variables and six equations.

In our case, evaluating the function $\mathbf{F}$ is relatively expensive. As a result, we want to use a gradient-based method, but we also would not like to have to evaluate the function seven times at each iteration. To alleviate this, we use Broyden's method, [15], which requires seven function evaluations to get the first estimate of the gradient matrix, $\partial \mathbf{F} / \partial \boldsymbol{v}$. Subsequent estimates are obtained using only the previous estimate of the gradient and the function evaluation of the current iteration.

\section{E. Linearization}

We can write our equations of motion as a generic nonlinear system

$$
\dot{\mathbf{x}}=\mathbf{F}(\mathbf{x}, \mathbf{u})
$$


For small perturbations from a trimmed flight condition, it is useful to study a linearized system of equations. To do this, we write

$$
\mathbf{x}=\overline{\mathbf{x}}+\Delta \mathbf{x} \quad \mathbf{u}=\overline{\mathbf{u}}+\Delta \mathbf{u}
$$

and substitute this into the original nonlinear system, Eq. (30). After taking a first-order Taylor series and canceling the trimmed part of the equations of motion, we get

$$
\Delta \dot{\mathbf{x}} \approx \mathbf{A} \Delta \mathbf{x}+\mathbf{B} \Delta \mathbf{u}
$$

Analysis of the linearized dynamics gives insight into the stability and control characteristics of the vehicle. The eigenvalues of $\mathbf{A}$ give the open-loop stability characteristics of the trimmed flight condition, and by selecting the controlled states and the corresponding control effectors to be used, we can also calculate the transmission zeros of the system.

The MASIV vehicle model often predicts that the thrust of the vehicle is not a smooth function of angle of attack and Mach number, [8]. Although the deviation from smoothness is only visible at small scales, this creates some difficulties in the estimation of the derivative matrices $\partial \mathbf{F} / \partial \mathbf{x}$ and $\partial \mathbf{F} / \partial \mathbf{u}$. Since these small-scale deviations are probably not physical, we chose to employ a method that reduces noise when calculating the derivative matrices. Rather than using a centered difference to calculate each derivative,

$$
\frac{\partial F_{j}}{\partial x_{k}} \approx \frac{F_{j}\left(\overline{\mathbf{x}}+\eta_{k} \mathbf{e}_{k}, \overline{\mathbf{u}}\right)-F_{j}\left(\overline{\mathbf{x}}-\eta_{k} \mathbf{e}_{k}, \overline{\mathbf{u}}\right)}{2 \eta_{k}}
$$

we use extra function evaluations (i.e. more than twice the number of state variables and control variables) and then fit a plane to the data using the method of linear least squares. This technique is essentially a low-pass filter, which reduces noise with high frequencies. The idea was formalized by Savitzky and Golay [16], although it has been around since the $19^{\text {th }}$ century [17].

\section{Results}

We present two categories of results in this section. The first part consists of results that pertain to the operating map of the vehicle over a significant portion of the flight envelope. The primary results consist of maps of the dependent trim variables across a range of Mach numbers and altitudes, but the effects of fuel mass, latitude, and flight path angle are also investigated. The second part of the results discusses the properties of the linearized equations of motion at several selected trimmed flight conditions.

In this paper, all results are for the vehicle shown in Figs. 1 and 2. The vehicle has two all-moving horizontal surfaces, which we refer to as elevators. There are also two rudders, although we do not allow them to move independently in these results. The inertia properties of the vehicle are given in Table 1. The mass estimates come from several correlations, which are given in [18]. The inertia tensor is calculated by assuming a constant density throughout the vehicle.

The effect of flying at a different latitude is shown in Fig. 8. One thing that is interesting about this comparison is that flight at $L=45^{\circ}$ requires that we utilize a full six-degree-of-freedom analysis, whereas flight above the equator can be analyzed using the longitudinal dynamics. This is because the Coriolis force due to rotating around the Earth's axis is not normal to the surface of the Earth.

Table 1. Inertia properties of example vehicle

\begin{tabular}{ccc}
\hline \hline Symbol & Name & Value \\
\hline & operational empty mass & $1.90 \times 10^{4} \mathrm{~kg}$ \\
& maximum fuel mass & $2.27 \times 10^{4} \mathrm{~kg}$ \\
$I_{x x}$ & specific moment of inertia & $2.45 \mathrm{~m}^{2}$ \\
$I_{y y}$ & specific moment of inertia & $34.93 \mathrm{~m}^{2}$ \\
$I_{z z}$ & specific moment of inertia & $36.34 \mathrm{~m}^{2}$ \\
$I_{x z}$ & specific product of inertia & $0.50 \mathrm{~m}^{2}$ \\
\hline \hline
\end{tabular}




\section{A. Operating Maps}

Figures $6,7,8$, and 9 show operating maps for five sets of vehicle conditions. For each set of vehicle conditions, a scram-mode trimmed flight condition was found (when possible) for altitudes ranging from 24 to $32 \mathrm{~km}$ and for Mach numbers ranging from 6 to 10. The first set of flight conditions, which is included in each figure for comparison, is for unaccelerated $(\dot{V}=0)$, level $(\gamma=0)$, eastward $\left(\chi=90^{\circ}\right)$ flight above the equator $(L=0)$ with an $50 \%$ fuel load. The sets of flight conditions present in each figure are explained in Table 2.

Table 2. Table of flight condition sets in Figs. $6,7,8$, and 9

\begin{tabular}{lccccc}
\hline \hline Description & Figures & Fuel & $L$ & $\gamma$ & $\chi$ \\
\hline Half fueled & $6,7,8,9$ & 0.5 & $0^{\circ}$ & $0^{\circ}$ & $90^{\circ}$ \\
Empty & 6 & 0.0 & $0^{\circ}$ & $0^{\circ}$ & $90^{\circ}$ \\
Fully fueled & 7 & 1.0 & $0^{\circ}$ & $0^{\circ}$ & $90^{\circ}$ \\
Mid latitude & 8 & 0.5 & $45^{\circ}$ & $0^{\circ}$ & $90^{\circ}$ \\
Climbing & 9 & 0.5 & $0^{\circ}$ & $5^{\circ}$ & $90^{\circ}$ \\
\hline \hline
\end{tabular}

Each figure consists of the operating maps for two configurations. In each case, the plots of the left (parts a, c, and e) are for the same reference configuration of the half-fueled vehicle in steady, level flight to the east at the equator. The plots on the right side of each figure (parts $b, d$, and f) show the operating map for one of the other four configurations. The top row (parts a and b) show the equivalence ratio for the trimmed flight condition, the second row (parts $\mathrm{c}$ and $\mathrm{d}$ ) show the trimmed angle of attack, and the bottom row (parts e and f) show the trim elevator deflection. In each plot, lines of constant dynamic pressure are plotted on top of the operating map. The values associated with each dynamic pressure contour are given in atmospheres. These lines of constant dynamic pressure serve two purposes: to show where lines of constant dynamic pressure are on the operating map and to suggest an upper limit on the Mach number for each altitude. The model used for these operating maps does not include an analysis of heat transfer, so the model predicts that the vehicle is able to trim at very high Mach numbers. However, a possible first approximation to the effect of heating considerations on the flight envelope is to limit the dynamic pressure to about 1 atmosphere.

In each of the operating maps, many of the same trends are present. The white area on the left of each plot shows the area in which the engine operates in ram mode. This is essentially the lower Mach number limit of the scram-mode flight envelope. The trends in equivalence ratio and angle of attack are probably the most interesting features of the plots. If we increase the Mach number while holding the altitude constant, the vehicle naturally gets more lift, so the trimmed angle of attack decreases. At the same time, the drag increases, so we need more thrust to maintain a constant Mach number. However, as the mass flow rate of air through the engine increases with increasing Mach number, the trimmed equivalence ratio actually decreases until the Mach number reaches about 9.0. The inlet used for this vehicle was designed to operate for Mach numbers between 7 and 9 using the methodology outlined in [9], and indeed we see sharp changes in the operating maps near Mach 9. For higher Mach numbers, the inlet behaves very poorly, and the drag increases very rapidly. The result is that the a large amount of fuel is needed for flight at higher Mach numbers.

Figures 6 and 7 focus on the effect of the mass of the vehicle on the operating map. As expected, when more fuel is present in the vehicle, the trimmed angle of attack increases in order to provide sufficient lift to account for the higher mass of the vehicle. While Fig. 7 shows that the trimmed flight conditions do not change much between the half-full and full operating conditions, there is a slight increase in angle of attack which leads to a relatively noticeable increase in fuel-air equivalence ratio. This essentially shows that a slight increase in angle of attack leads to a significant increase in drag or a significant decrease in thrust. The difference between the half-fueled and empty configurations, as shown in Fig. 6, is more pronounced. The vehicle is able to trim at a significantly lower angle of attack, and the ram/scram transition boundary tends to occur at a slightly lower Mach number. The reason for this difference is probably that the ratio of the half-empty mass to the empty mass is higher than the ratio of the full mass to the half-empty mass.

The effect of flying at a different latitude is shown in Fig. 8. One thing to note about this comparison is that flight at $L=45^{\circ}$ requires a full six-degree-of-freedom model to trim the vehicle, whereas flight over the equator does not. When flying east or west above the equator, the Coriolis force due to the Earth's rotation and the vehicle's movement around the Earth both point directly normal to the Earth's surface, and we know that keeping $\Phi, \delta_{D E}$, and $\delta_{C R}$ all equal to zero will ensure that $\dot{V}, \dot{P}$, and $\dot{R}$ are all zero. Therefore, we can use the longitudinal equations of motion without any loss of accuracy. For other values of latitude and heading angle, this is not true. However, it is interesting to note that the operating map is barely affected at all by this change in latitude. In the $L=45^{\circ}$ analysis, we have 
trimmed the flight condition for $\dot{U}, \dot{V}, \dot{W}, \dot{P}, \dot{Q}$, and $\dot{R}$ all equaling zero. This means that the vehicle is not traveling along a great circle, but is flying at constant latitude. In addition, the vehicle has to fly at a noticeable roll angle $(\Phi$ ranging from $-4.5^{\circ}$ to $-12^{\circ}$ throughout the operating maps in Fig. 8b, d, and f) to counteract the component of the Coriolis force that points south. Despite these two differences, the remaining properties of the operating maps are very similar.

Figure 9 shows operating maps for the vehicle in climbing flight. We held $\gamma=5^{\circ}$ constant, which does mean that the climb rate, $-V_{D}$, varies as a function of Mach number and altitude. The minimum and maximum values of the climb rate are $155 \mathrm{~m} / \mathrm{s}$ and $265 \mathrm{~m} / \mathrm{s}$, respectively. It may come as a surprise that the angle of attack is actually lower for climbing flight, but this can be explained in terms of some of the weight of the vehicle now being in the $-x^{b}$ direction, which means that more thrust and less lift is needed. In Fig. $9 a$ and $b$, we do see that the trimmed equivalence ratio is significantly higher for climbing flight.

The final comment in this section is on the need or lack thereof of a rotating ellipsoidal Earth model. The MASTrim software package contains options to use a variety of models, so we were able to easily test the importance of the Earth's shape and rotation separately. Table 3 shows the trimmed flight conditions using four different models. A flight condition at a middle latitude was selected because the ellipsoidal and spherical models are equivalent at the equator (when flying directly east or west), and it also introduces a nonzero roll angle. The spherical models can be obtained from the equations of motion in Section III by setting $e=0$, and the nonrotating-Earth model additionally sets $\omega_{i e}$ equal to zero. The flat-Earth model makes the further assumption that $r_{Q}$ goes to infinity, and the vectors $\boldsymbol{\omega}_{e n}^{n}$ and $\dot{\omega}_{e n}^{n}$ are zero, as well. We can see from the table of trimmed conditions that the flat-Earth model makes significant errors. The nonrotating-Earth model is qualitatively close, but it eliminates the dependence of the equations of motion on the heading angle. Finally, we see that using a rotating, spherical Earth does not introduce significant errors. This is expected since the Earth is nearly spherical, although it does add the potential of navigational inaccuracy if we are considering a trajectory between two points.

Table 3. Comparison of trimmed flight conditions for half-fueled vehicle flying east at Mach 8 , an altitude of $26 \mathrm{~km}$, and at $45^{\circ} \mathrm{N}$ latitude.

\begin{tabular}{lcccc}
\hline \hline Earth model & $\delta_{E R}$ & $\alpha$ & $\delta_{C E}$ & $\Phi$ \\
\hline Rotating, ellipsoidal Earth & 0.2556 & $0.0370^{\circ}$ & $2.6846^{\circ}$ & $-7.5644^{\circ}$ \\
Rotating, spherical Earth & 0.2554 & $0.0343^{\circ}$ & $2.6857^{\circ}$ & $-7.5897^{\circ}$ \\
Nonrotating, spherical Earth & 0.2588 & $0.0710^{\circ}$ & $2.6711^{\circ}$ & $-5.7840^{\circ}$ \\
Flat Earth & 0.2719 & $0.2208^{\circ}$ & $2.5219^{\circ}$ & $0^{\circ}$ \\
\hline \hline
\end{tabular}

\section{B. Linearized Motion Properties}

We performed a linearization of the equations of motion at three flight conditions. The selected trimmed flight conditions are all for the half-fueled vehicle, using unaccelerated, level flight to the east at an altitude of $26 \mathrm{~km}$. The first case, for which the pole/transmission zero maps are shown in Fig. 10, is for a Mach number of 8.0 and at the equator. The second case, which is shown in Fig. 11, is for a flight Mach number of 9.0. Finally, Fig. 12 shows the poles and transmission zeros for flight at a latitude of $45^{\circ} \mathrm{N}$. The reference trimmed flight conditions, about which the linear systems are obtained, are given in full detail in Table 4.

We used

$$
\mathbf{x}=\left[\begin{array}{llllllllll}
h & V_{t} & \alpha & \beta & \Phi & \Theta & \Psi & P & Q & R
\end{array}\right]^{T}
$$

as the full set of state variables, and the full set of inputs was

$$
\mathbf{u}=\left[\begin{array}{llll}
\delta_{E R} & \delta_{C E} & \delta_{D E} & \delta_{C R}
\end{array}\right]^{T}
$$

Finally, the outputs were selected to be

$$
\mathbf{y}=\left[\begin{array}{llll}
V_{t} & \gamma & \chi & \Phi
\end{array}\right]^{T}
$$

The states were split into longitudinal states of $h, V_{t}, \alpha, \Theta$, and $Q$ and lateral states of $\beta, \Phi, \Psi, P$, and $R$. The longitudinal inputs are $\delta_{E R}$ and $\delta_{C E}$, and the lateral inputs are $\delta_{D E}$ and $\delta_{C R}$. Finally, the outputs were split into longitudinal outputs, $V_{t}$ and $\gamma$, and lateral outputs, $\chi$ and $\Phi$. 


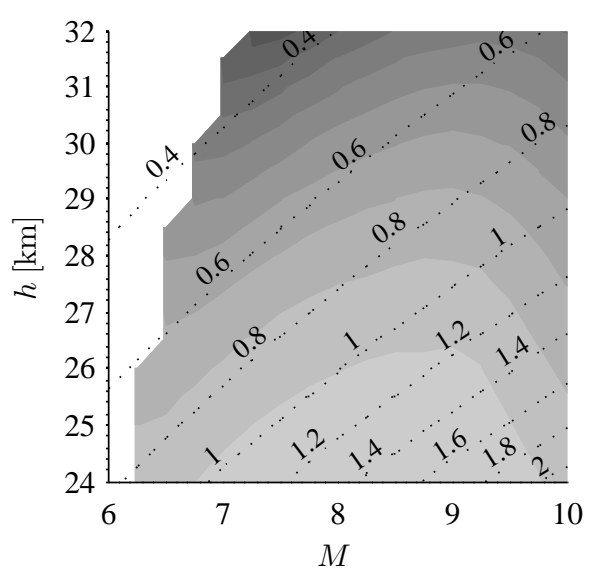

a) $\delta_{E R}$, half full

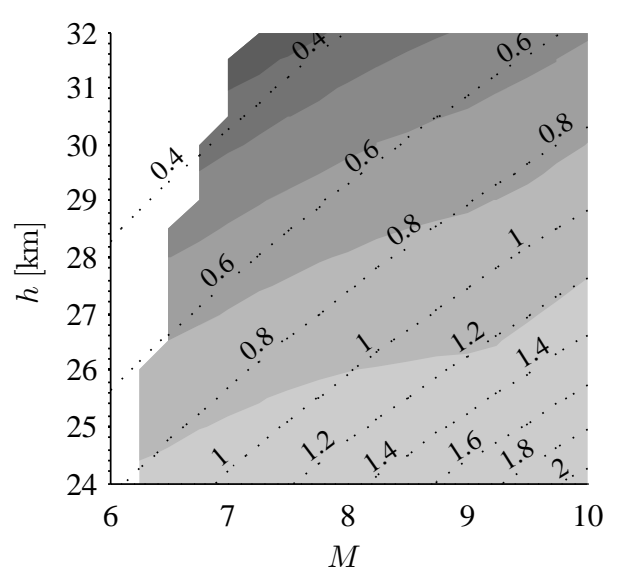

c) $\alpha$ (degrees), half full

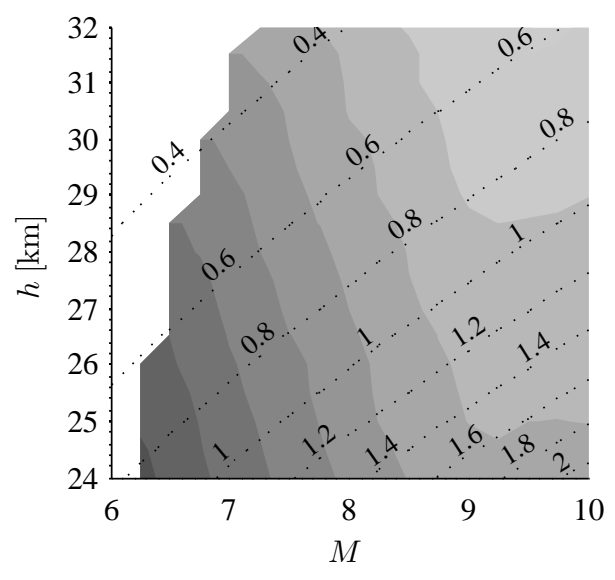

e) $\delta_{C E}$ (degrees), half full
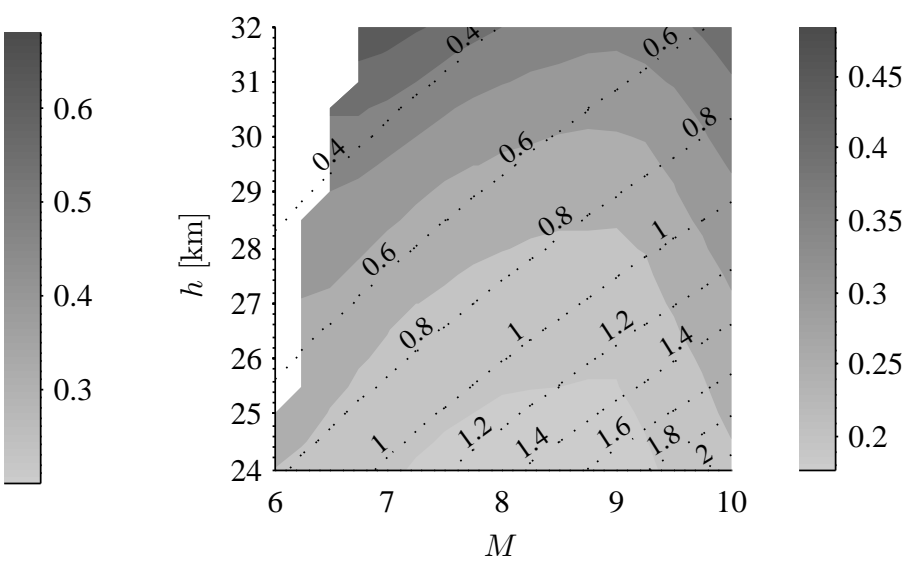

b) $\delta_{E R}$, empty
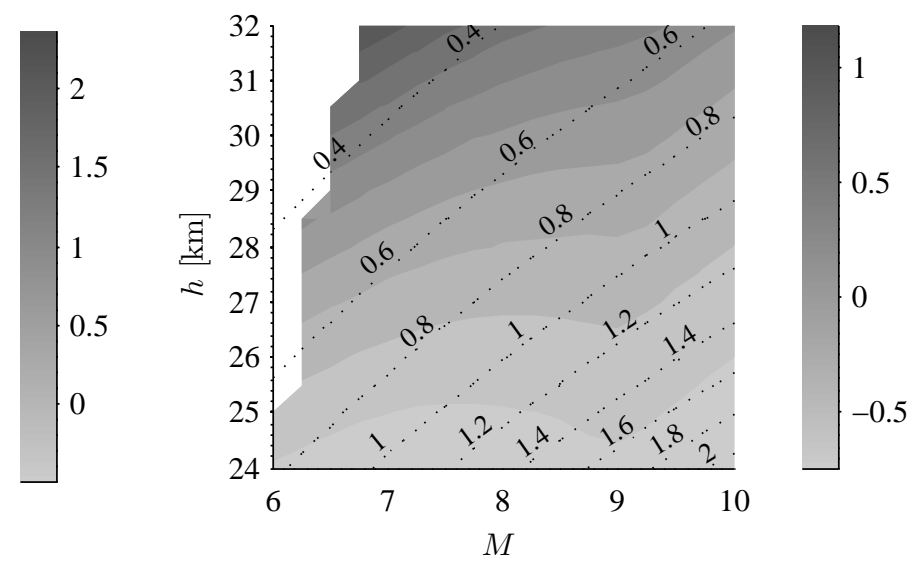

d) $\alpha$ (degrees), empty
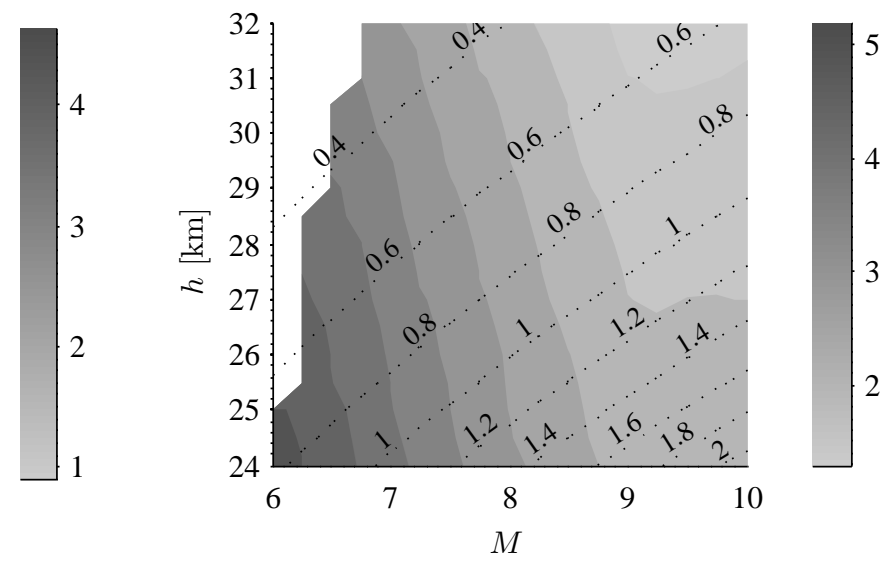

f) $\delta_{C E}$ (degrees), empty

Figure 6. Comparison of operating maps between empty and full configurations for level flight to the east at the equator. Dotted lines show contours of dynamic pressure in atmospheres. 


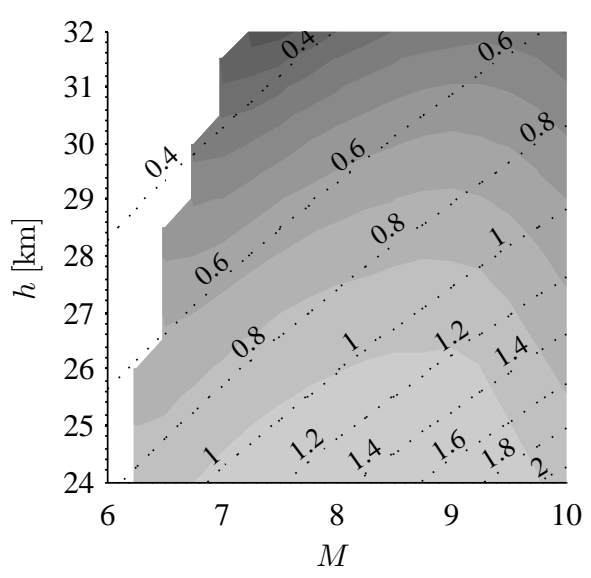

a) $\delta_{E R}$, half full

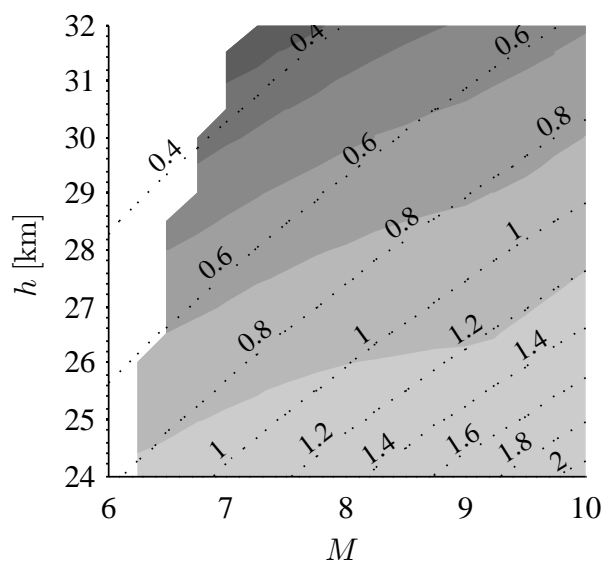

c) $\alpha$ (degrees), half full

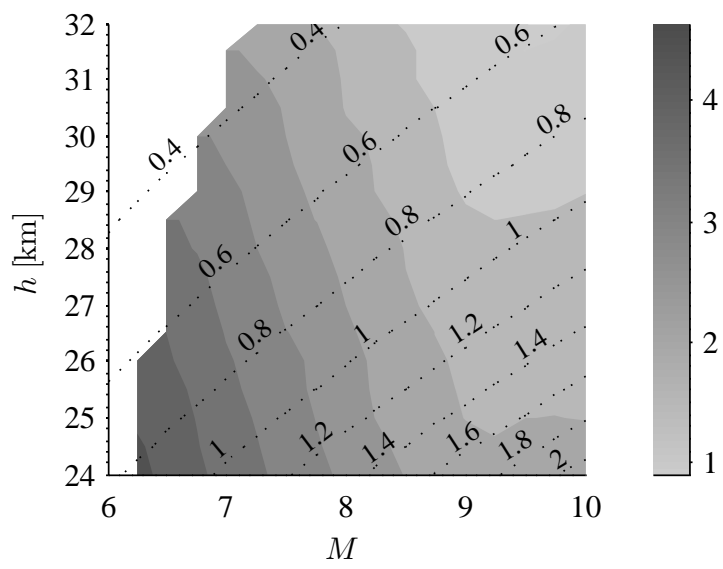

e) $\delta_{C E}$ (degrees), half full

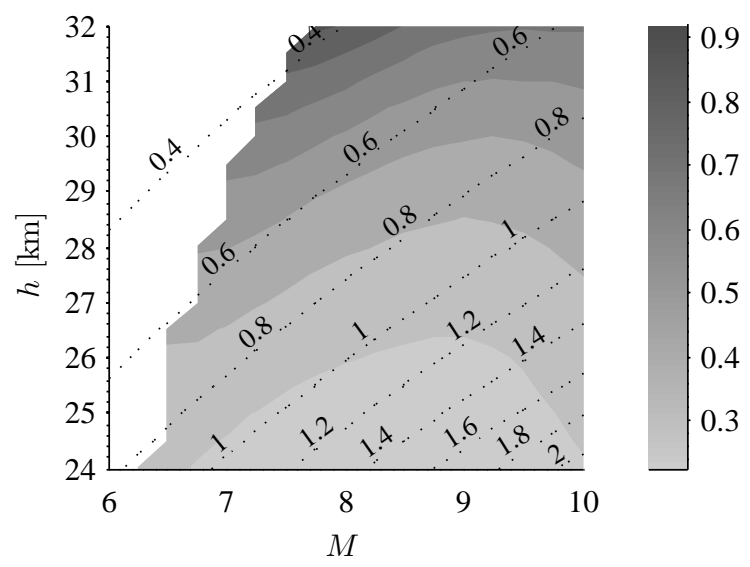

b) $\delta_{E R}$, full

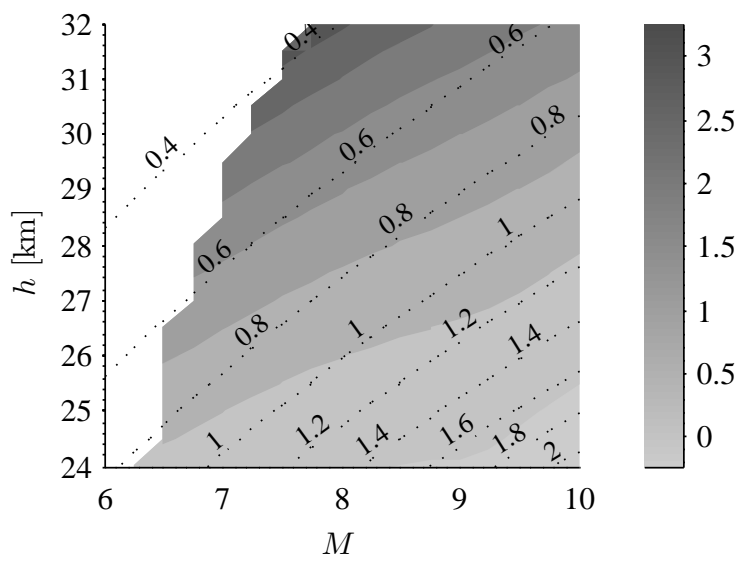

d) $\alpha$ (degrees), full

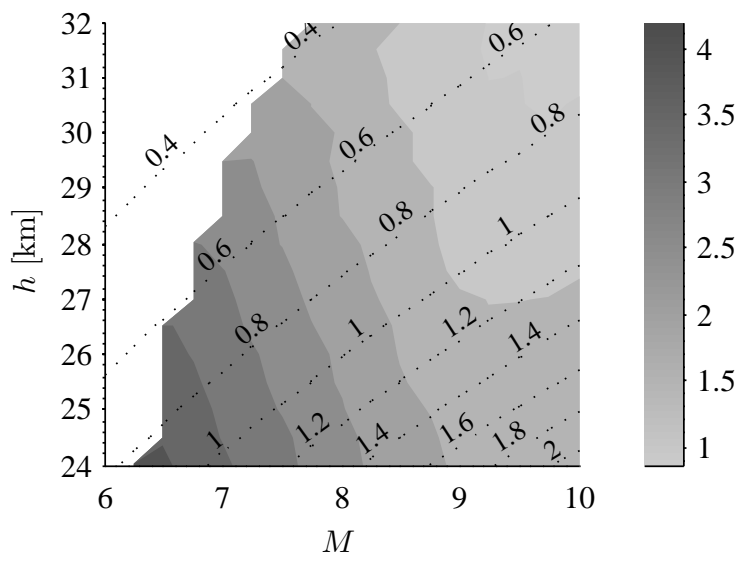

f) $\delta_{C E}$ (degrees), full

Figure 7. Comparison of operating maps between fully fueled and half fueled configurations for level flight to the east at the equator. Dotted lines show contours of dynamic pressure in atmospheres. 


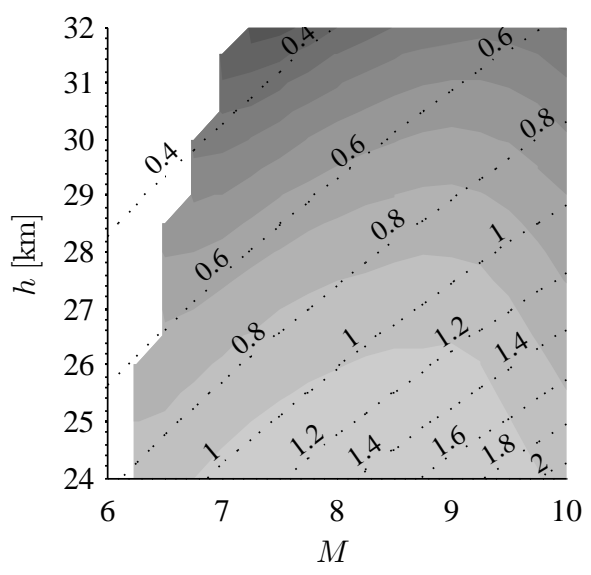

a) $\delta_{E R}, L=0^{\circ}$

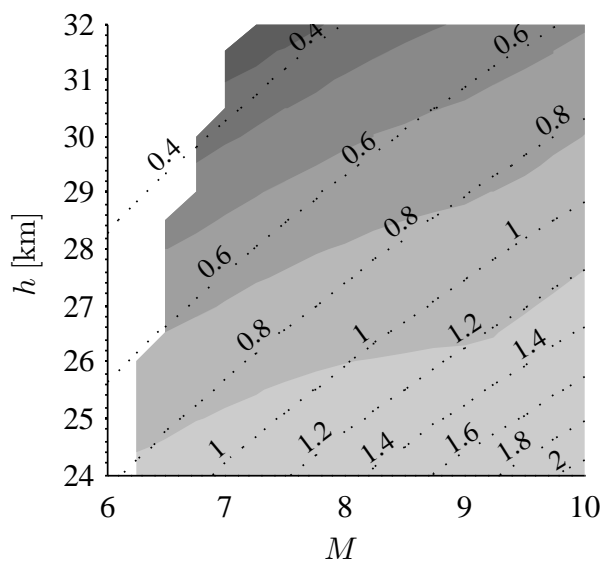

c) $\alpha$ (degrees), $L=0^{\circ}$

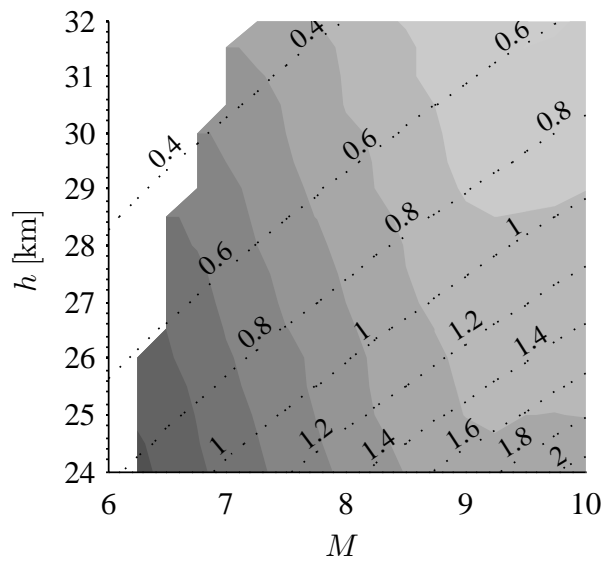

e) $\delta_{C E}$ (degrees), $L=0^{\circ}$
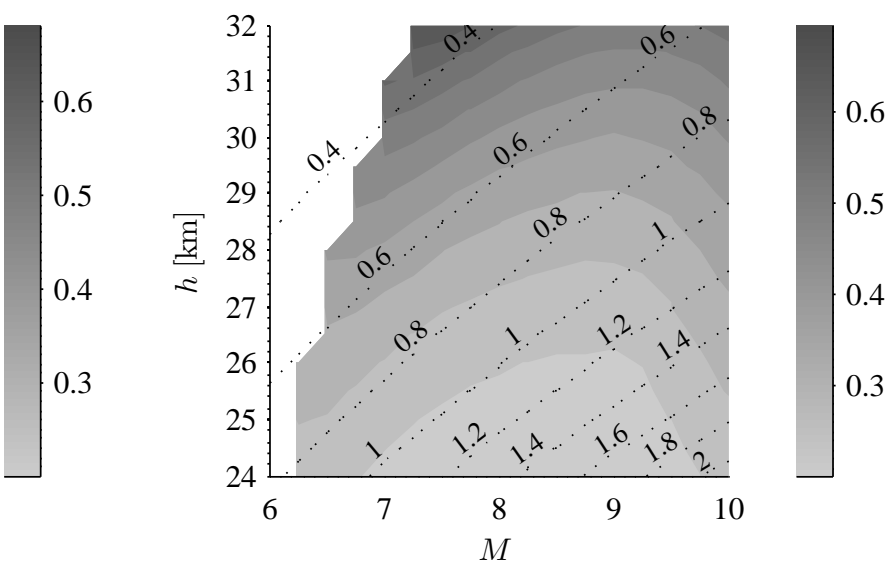

b) $\delta_{E R}, L=45^{\circ}$
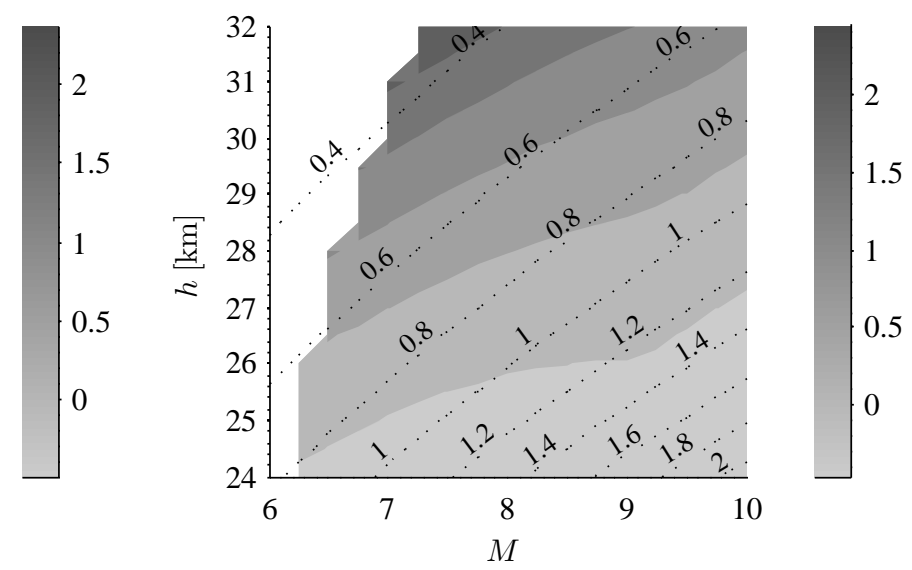

d) $\alpha$ (degrees), $L=45^{\circ}$
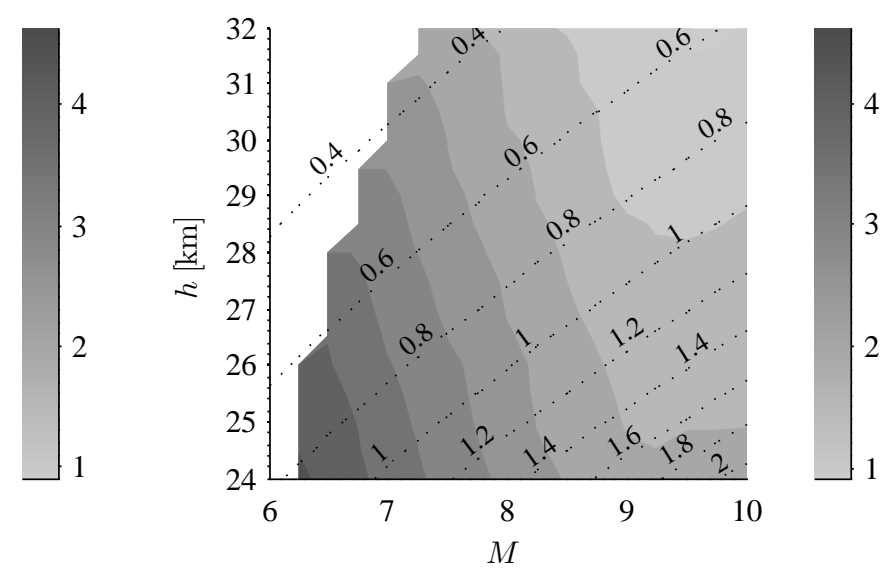

f) $\delta_{C E}$ (degrees), $L=45^{\circ}$

Figure 8. Comparison of operating maps for half-fueled configuration for level, eastward flight at two different latitudes. Dotted lines show contours of dynamic pressure in atmospheres. 


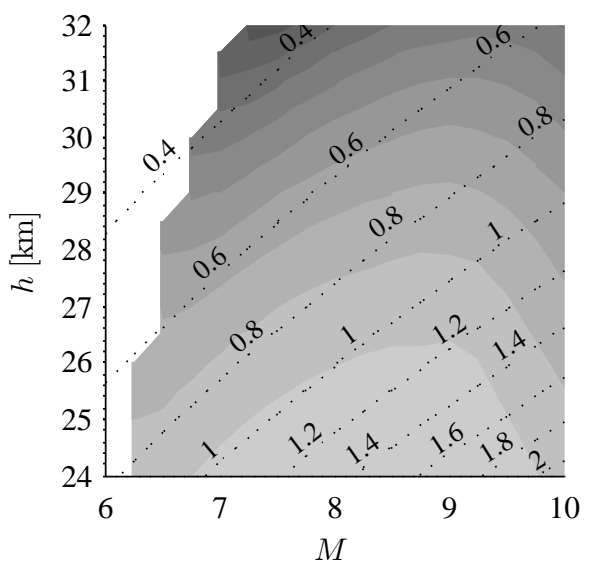

a) $\delta_{E R}, \gamma=0^{\circ}$

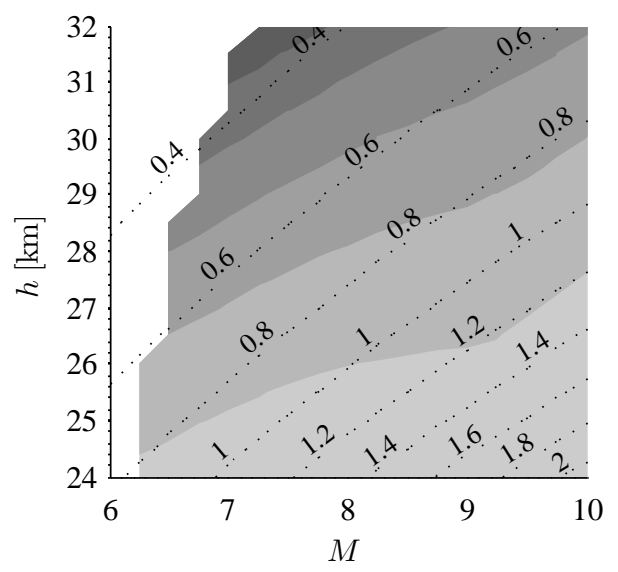

c) $\alpha$ (degrees), $\gamma=0^{\circ}$

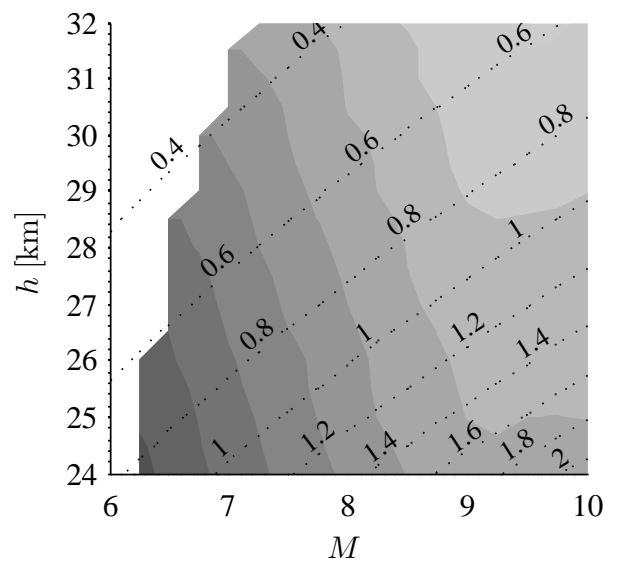

e) $\delta_{C E}$ (degrees), $\gamma=0^{\circ}$
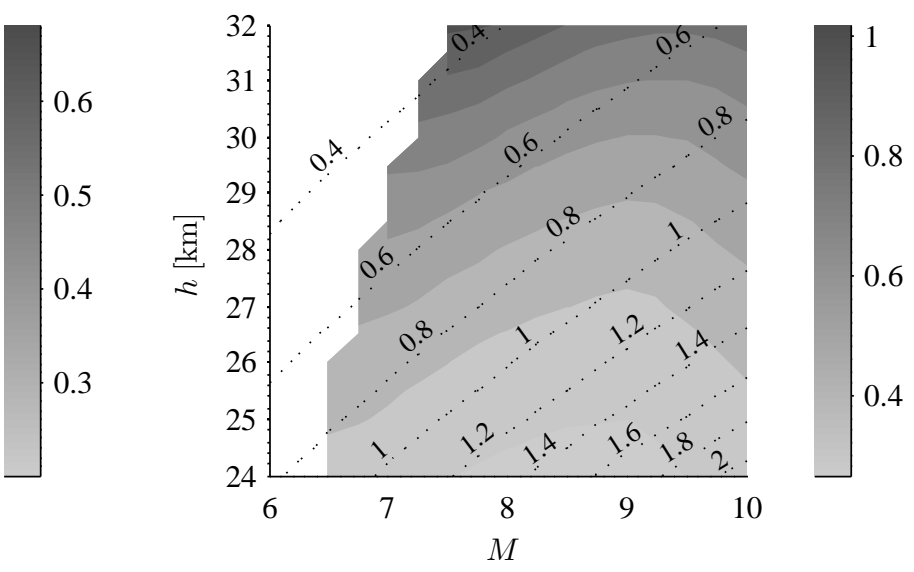

b) $\delta_{E R}, \gamma=5^{\circ}$
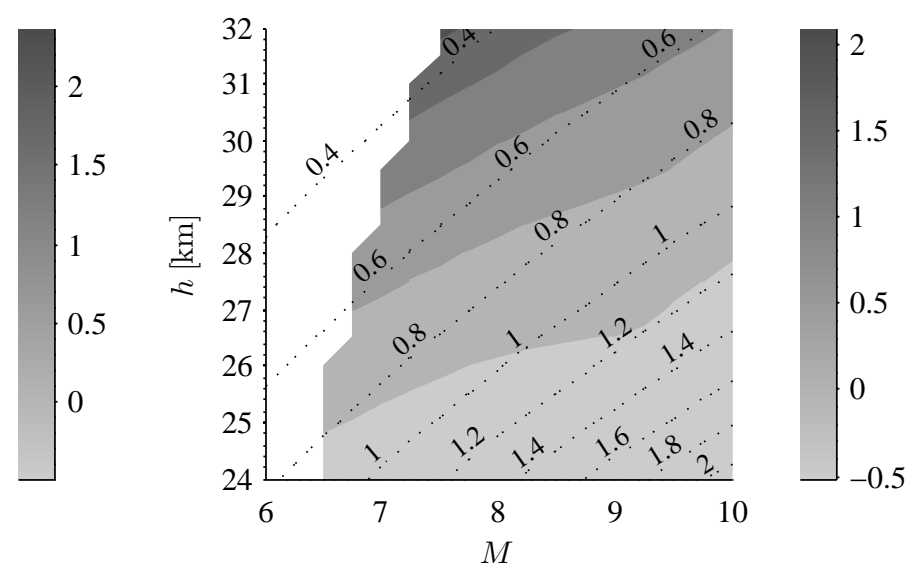

d) $\alpha$ (degrees), $\gamma=5^{\circ}$
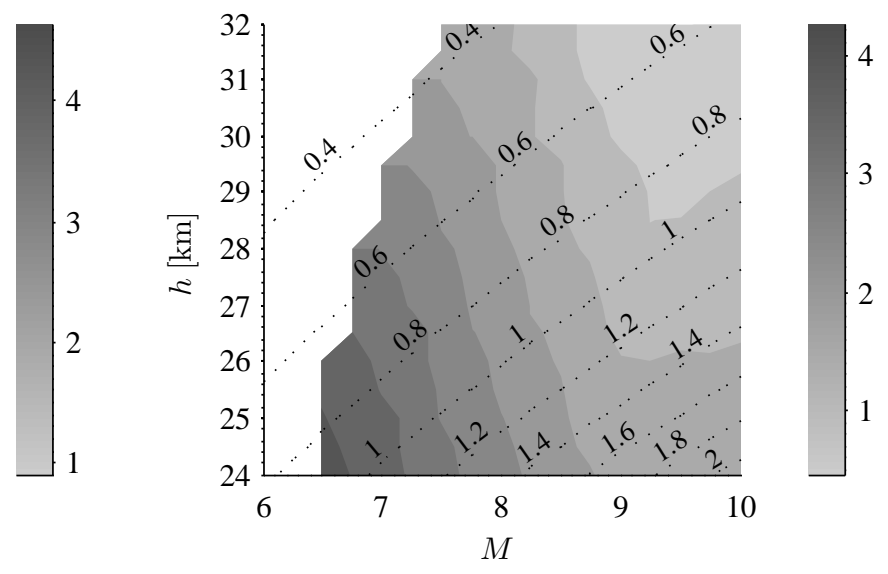

f) $\delta_{C E}$ (degrees), $\gamma=5^{\circ}$

Figure 9. Comparison of operating maps for level and climbing conditions for the half-fueled vehicle flying eastward over the equator. Dotted lines show contours of dynamic pressure in atmospheres. 


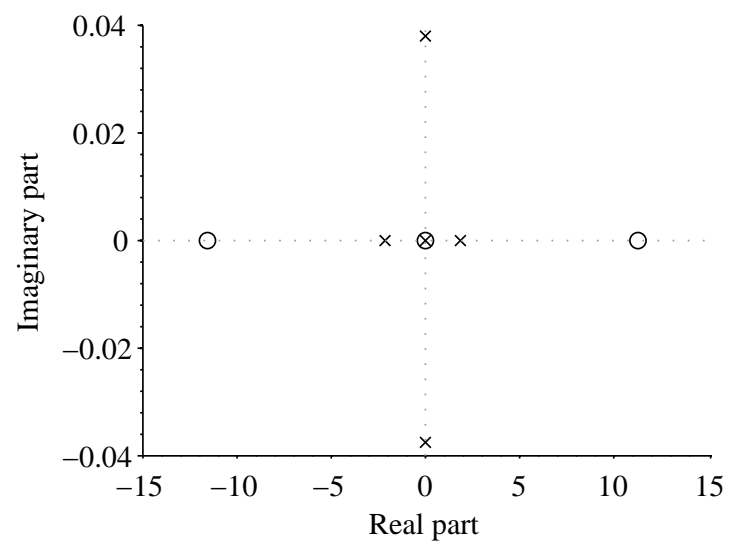

a) Longitudinal dynamics

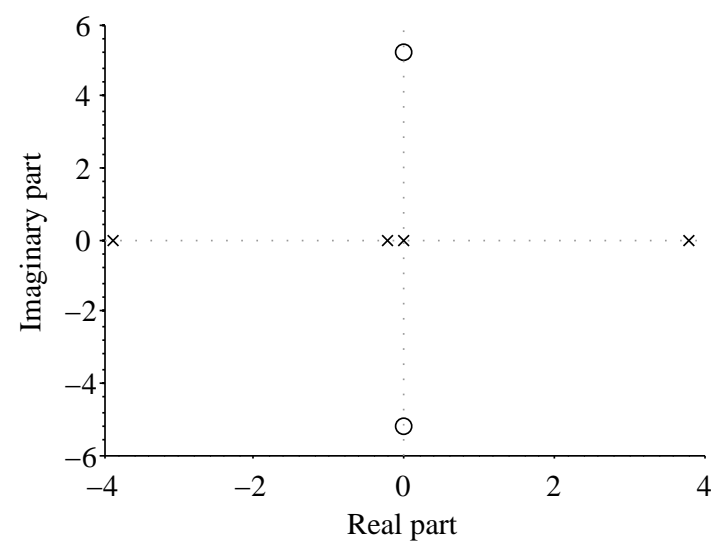

b) Lateral dynamics

Figure 10. Pole/transmission zero maps of the Jacobian linearization about the trimmed Mach 8 flight condition

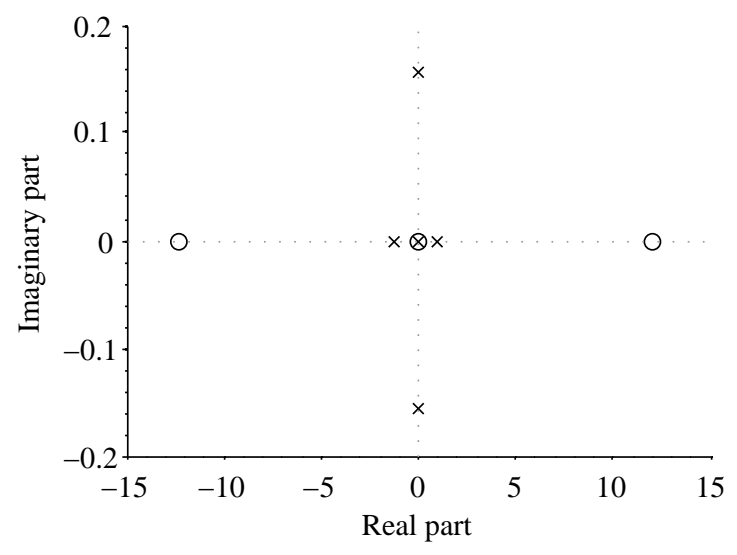

a) Longitudinal dynamics

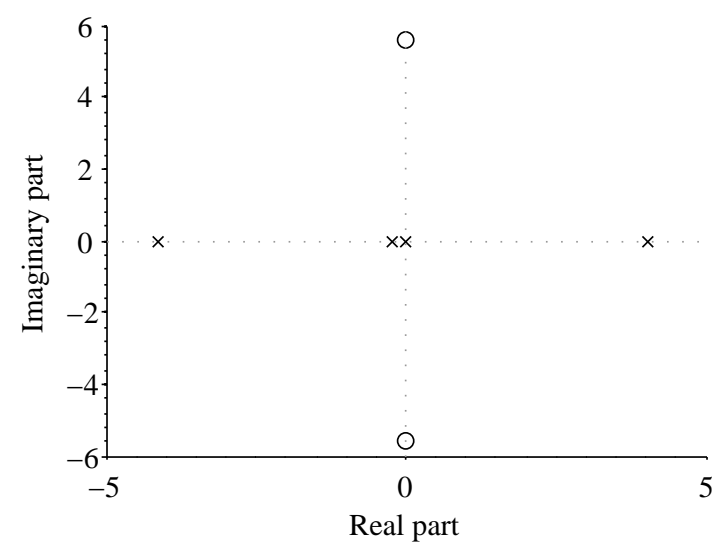

b) Lateral dynamics

Figure 11. Pole/transmission zero maps of the Jacobian linearization about the trimmed Mach 9 flight condition

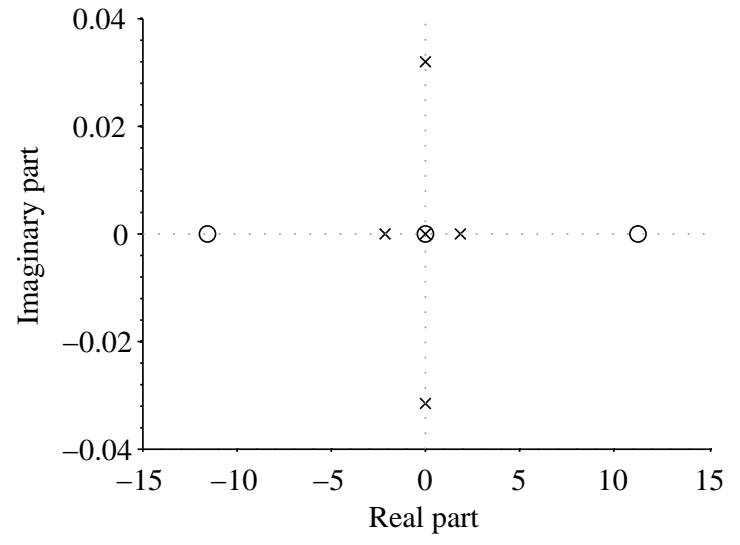

a) Longitudinal dynamics

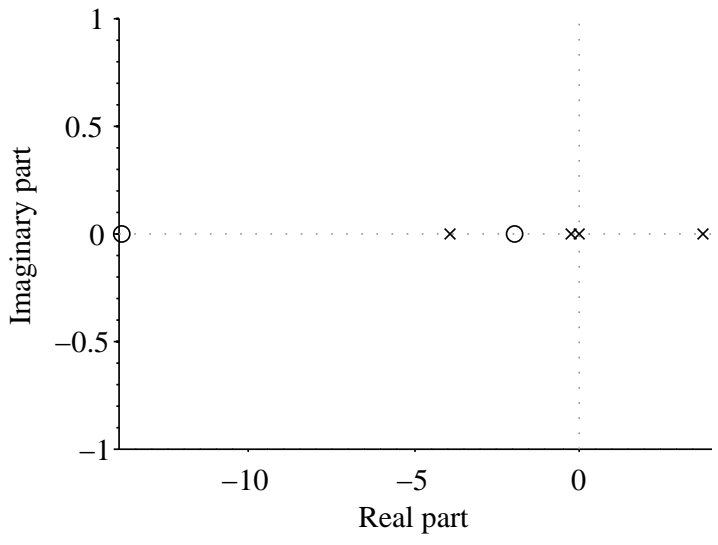

b) Lateral dynamics

Figure 12. Pole/transmission zero maps of the Jacobian linearization about the trimmed Mach 8 flight condition at a latitude of $45^{\circ} \mathrm{N}$

18 of 22 
Table 4. Trim conditions for the linearizations

\begin{tabular}{cccc}
\hline \hline State or input & Fig. $10, M=8.0$ & Fig. $11, M=9.0$ & Fig. $12, L=45^{\circ}$ \\
\hline$h$ & $26 \mathrm{~km}$ & $26 \mathrm{~km}$ & $26 \mathrm{~km}$ \\
$V_{t}$ & $2393.6679 \mathrm{~m} / \mathrm{s}$ & $2692.8764 \mathrm{~m} / \mathrm{s}$ & $2393.6679 \mathrm{~m} / \mathrm{s}$ \\
$\alpha$ & $0.0079^{\circ}$ & $-0.0447^{\circ}$ & $0.0370^{\circ}$ \\
$\beta$ & $0^{\circ}$ & $0^{\circ}$ & $0^{\circ}$ \\
$\Phi$ & $0^{\circ}$ & $0^{\circ}$ & $-7.6544^{\circ}$ \\
$\Psi$ & $90^{\circ}$ & $90^{\circ}$ & $89.9951^{\circ}$ \\
$\Theta$ & $0.0079^{\circ}$ & $-0.0447^{\circ}$ & $0.0367^{\circ}$ \\
$P$ & $0^{\circ} / \mathrm{s}$ & $0^{\circ} / \mathrm{s}$ & $0^{\circ} / \mathrm{s}$ \\
$Q$ & $0^{\circ} / \mathrm{s}$ & $0^{\circ} / \mathrm{s}$ & $0^{\circ} / \mathrm{s}$ \\
$R$ & $0^{\circ} / \mathrm{s}$ & $0^{\circ} / \mathrm{s}$ & $0^{\circ} / \mathrm{s}$ \\
\hline$\delta_{E R}$ & 0.2526 & 0.2404 & 0.2556 \\
$\delta_{C E}$ & $2.6744^{\circ}$ & $1.8940^{\circ}$ & $2.6846^{\circ}$ \\
$\delta_{D E}$ & $0^{\circ}$ & $0^{\circ}$ & $0^{\circ}$ \\
$\delta_{D R}$ & $0^{\circ}$ & $0^{\circ}$ & $0^{\circ}$ \\
\hline \hline
\end{tabular}

The change due to increasing the Mach number, which can be inspected from the differences and similarities of Figs. 10 and 11, appears to be very slight. In all three cases, the longitudinal dynamics, shown in Figs. 10a, 11a, and 12a, exhibit similar characteristics.

The lateral dynamics, shown in Figs. 10b, 11b, and 12b, show slightly more variance. In all three cases, the poles are quite similar, and the main trend appears to be that increasing the Mach number shortens the period of each mode. However, the zeros are significantly different for the case of flight at $L=45^{\circ}$. The main reason for this is that the roll state, $\Phi$, is not zero in this case. The result is increased control authority of the heading output, $\chi$. Since roll is the most effective way to control heading, the response of the system to small changes in roll is important in determining how easily $\chi$ can be controlled. However, when $\Phi$ is zero, small changes in $\Phi$ result in only small changes in the lateral force.

In all three cases, one of the zeros of the pitch dynamics is nonminimum phase, which is consistent with the results in [4]. This is expected because of the actions the vehicle needs to take to increase $\gamma$. In order to increase the climb rate, the vehicle needs to deflect the elevators upward to increase the angle of attack, but deflecting the elevators decreases the lift instantaneously, which causes the aircraft to plunge and drop altitude before it begins to pitch nose-up and increase $\alpha$ and thus lift. These nonminimum phase dynamics affect the control design, and are important in this case because the vehicle is open-loop unstable, and the transmission zeros effectively limit the amount of bandwidth in the system, thus affecting performance.

Table 5. Longitudinal real poles and time-to-double or half-amplitude $\left(L=45^{\circ} \mathrm{N}, \chi=90^{\circ}, M=8, h=26 \mathrm{~km}\right)$

\begin{tabular}{cc}
\hline \hline Pole & Time to double \\
\hline-2.24 & 0.31 \\
1.845 & 0.38 \\
-0.0058 & 120.0 \\
\hline \hline
\end{tabular}

Table 6. Longitudinal complex-conjugate poles, damping ratios, and natural frequencies $\left(L=45^{\circ} \mathrm{N}, \chi=90^{\circ}, M=8, h=26 \mathrm{~km}\right)$

\begin{tabular}{ccc}
\hline \hline Pole & Damping ratio & Natural frequency \\
\hline$-6.78 \times 10^{-4} \pm 4.80 \times 10^{-2} j$ & $1.41 \times 10^{-2}$ & $4.80 \times 10^{-2}$ \\
\hline \hline
\end{tabular}

Tables 5, 6, and 7 give a detailed analysis of the poles from Fig. 10. Presented in Table 8 is the contribution of each state to a given eigenvalue [19]. Each row corresponds to a state and each column corresponds to an eigenvalue 
Table 7. Lateral-directional real poles and time-to-double or half-amplitude $\left(L=45^{\circ} \mathrm{N}, \chi=90^{\circ}, M=8, h=26 \mathrm{~km}\right)$

\begin{tabular}{cc}
\hline \hline Pole & Time to double \\
\hline 3.83 & 0.18 \\
-3.87 & 0.18 \\
-0.28 & 2.50 \\
-0.0029 & 240.0 \\
\hline \hline
\end{tabular}

Table 8. State participation in eigenvalues of linearized system $\left(L=45^{\circ} \mathrm{N}, \chi=90^{\circ}, M=8, h=26 \mathrm{~km}\right)$

\begin{tabular}{lcccccccc}
\hline \hline & -3.87 & -2.24 & 3.83 & 1.84 & -0.28 & $-0.0007 \pm 0.05 j$ & -0.0058 & -0.0029 \\
\hline$\Delta h$ & 0.00 & 0.00 & 0.00 & 0.00 & 0.00 & 0.45 & 0.11 & 0.00 \\
$\Delta V_{t}$ & 0.00 & 0.00 & 0.00 & 0.00 & 0.00 & 0.05 & 0.89 & 0.00 \\
$\Delta \alpha$ & 0.00 & 0.43 & 0.00 & 0.45 & 0.00 & 0.06 & 0.00 & 0.00 \\
$\Delta \beta$ & 0.49 & 0.00 & 0.51 & 0.00 & 0.00 & 0.00 & 0.00 & 0.00 \\
$\Delta \Phi$ & 0.00 & 0.00 & 0.00 & 0.00 & 0.05 & 0.00 & 0.00 & 0.94 \\
$\Delta \Theta$ & 0.00 & 0.00 & 0.00 & 0.00 & 0.00 & 0.50 & 0.00 & 0.00 \\
$\Delta P$ & 0.00 & 0.00 & 0.00 & 0.00 & 0.99 & 0.00 & 0.00 & 0.01 \\
$\Delta Q$ & 0.00 & 0.51 & 0.00 & 0.49 & 0.00 & 0.00 & 0.00 & 0.00 \\
$\Delta R$ & 0.46 & 0.00 & 0.44 & 0.00 & 0.05 & 0.00 & 0.00 & 0.06 \\
\hline \hline
\end{tabular}

of the linearized dynamics. Each row and column of the table are normalized and must sum to 1 (or approximately 1 allowing for truncation error). For example, columns 2 and 4 are dominated by $\Delta \alpha$ and $\Delta Q$, so this is the short period mode, and this tells us that the standard two-state approximation is valid. Likewise columns 1 and 3 are the Dutch-roll mode $(\Delta \beta$ and $\Delta R)$. The spiral mode and roll modes are as we would expect, each being dominated by $\Delta \Phi$ and $\Delta P$, respectively.

More interesting are the columns with the complex-conjugate pair (column 6) and column 7 of the table. Taken together, these modes are a combination of the phugoid and what is often called an "altitude" mode. However, inspection of the sensitivities show that the slow, stable, real pole at -0.0058 is really dominated by velocity and to a lesser extent has an altitude component that cannot be neglected. Given the participation of the altitude in this particular eigenvalue, and likewise a small participation of velocity in the complex-conjugate pair, the interpretation here is that the proper approximation of this mode requires $\Delta h, \Delta \Theta$, and $\Delta V_{t}$.

\section{Conclusion}

In this paper, we explored a control-oriented model of an air-breathing hypersonic vehicle. The model includes predictions for wave interactions, fuel mixing, and finite-rate chemistry. This framework has been integrated into a three-dimensional vehicle design and a rotating, ellipsoidal Earth model for the equations of motion. Using this combined model, we determined more than 1400 trimmed flight conditions for various configurations of the vehicle. The parameters that were varied in this investigation were Mach number, altitude, amount of fuel, latitude, and flightpath angle. We also investigated the linearized dynamics for three of these trimmed flight conditions.

The main analysis presented in this paper for the various trim conditions was operating maps showing the appropriate equivalence ratio, angle of attack, and elevator angle for trimmed flight as a function of Mach number and altitude. These operating maps reveal important properties of the vehicle's performance, such as the Mach number at which ram-scram transition occurs and how the vehicle reacts to flying at a Mach number above the inlet design specifications. This type of performance analysis, and the modest computational resources required to do it, provides a useful tool during the conceptual design phase of the vehicle design process. 


\section{Acknowledgments}

This research is funded by the Air Force Research Laboratory/Air Vehicles Directorate grant FA 8650-07-2-3744 for the Michigan/AFRL Collaborative Center in Control Sciences (Michael Bolender as technical monitor).

Approved for Public Release; Distribution Unlimited. Case Number 88ABW-2011-1250.

\section{References}

[1] Schöttle, U. M. and Hillesheimer, M., "Performance Optimization of an Airbreathing Launch Vehicle by a Sequential Trajectory Optimization and Vehicle Design Scheme," AIAA Guidance, Navigation and Control Conference, 1991, AIAA Paper 91-2655. 2

[2] Chavez, F. R. and Schmidt, D. K., "Analytical Aeropropulsive/Aeroelastic Hypersonic-Vehicle Model with Dynamic Analysis," Journal of Guidance, Control, and Dynamics, Vol. 17, No. 6, 1994, pp. 1308-1319. 2

[3] Bolender, M. A. and Doman, D. B., "Nonlinear Longitudinal Dynamical Model of an Air-Breathing Hypersonic Vehicle," Journal of Spacecraft and Rockets, Vol. 44, No. 2, 2007, pp. 374-387. 2

[4] Parker, J. T., Serrani, A., Yurkovich, S., Bolender, M. A., and Doman, D. B., "Control-Oriented Modeling of an Air-Breathing Hypersonic Vehicle," Journal of Guidance, Control, and Dynamics, Vol. 30, No. 3, 2007, pp. 856869. 2,19

[5] Frendreis, S. G. V. and Cesnik, C. E. S., "3D Simulation of a Flexible Hypersonic Vehicle," Atmospheric Flight Mechanics Conference \& Exhibit, 2010, AIAA Paper 2010-8229. 2

[6] Dalle, D. J., Fotia, M. L., and Driscoll, J. F., "Reduced-Order Modeling of Two-Dimensional Supersonic Flows with Applications to Scramjet Inlets," Journal of Propulsion and Power, Vol. 26, No. 3, 2010, pp. 545-555. 2, 4

[7] Dalle, D. J., Frendreis, S. G. V., Driscoll, J. F., and Cesnik, C. E. S., "Hypersonic Vehicle Flight Dynamics with Coupled Aerodynamics and Reduced-order Propulsive Models," AIAA Atmospheric Flight Mechanics Conference \& Exhibit, 2010, AIAA Paper 2010-7930. 2, 4

[8] Torrez, S. M., Driscoll, J. F., Dalle, D. J., Bolender, M. A., and Doman, D. B., "Hypersonic Vehicle Thrust Sensitivity to Angle of Attack and Mach Number," AIAA Atmospheric Flight Mechanics Conference, 2009, AIAA Paper 2009-6152. 2, 11

[9] Torrez, S. M., Driscoll, J. F., Dalle, D. J., and Fotia, M. L., "Preliminary Design Methodology for Hypersonic Engine Flowpaths," 16th AIAA/DLR/DGLR International Space Planes and Hypersonic Systems and Technologies Conference, 2009, AIAA Paper 2009-7289. 2, 4, 12

[10] Hall, K. C., Thomas, J. P., and Dowell, E. H., "Proper Orthogonal Decomposition Technique for Transonic Unsteady Aerodynamic Flows," AIAA Journal, Vol. 38, No. 10, 2000, pp. 1853-1862. 3

[11] White, F. M., Viscous Fluid Flow, McGraw-Hill, 3rd ed., 2006. 4

[12] Van Driest, E. R., “The Problem of Aerodynamic Heating," Aeronautical Engineering Review, Vol. 15, No. 10, 1956, pp. 26-41. 4, 5

[13] Anon., "Department of Defense World Geodetic System 1984," Tech. Rep. TR8350.2, 3rd ed., National Imagery and Mapping Agency (now NGA), 1997. 7, 9

[14] Groves, P. D., Principles of GNSS, Inertial, and Multisensor Integrated Navigations Systems, Artec House, 2008. 7,9

[15] Broyden, C. G., "A Class of Methods for Solving Nonlinear Simultaneous Equations," Mathematics of Computation, Vol. 19, No. 92, October 1965, pp. 577-593. 10

[16] Savitzky, A. and Golay, M. J. E., "Smoothing and Differentiation of Data by Simplified Least Squares Procedures," Analytical Chemistry, Vol. 36, No. 8, 1964, pp. 1627-1639. 11

[17] Whittaker, S. E. and Robinson, G., The Calculus of Observations, Blackie \& Son, Limited, 1924. 11

21 of 22 
[18] Chudoba, B., "Aircraft Volume and Mass Guidelines," Tech. rep., National Institute of Aerospace, June 2008, Hypersonic Educational Initiative Hypersonic Vehicle System Integration Short Course. 11

[19] Durham, B., "Aircraft Dynamics \& Control," http://www. aoe.vt.edu/ durham/AOE5214, accessed March 3, 2011. 19 